\title{
LA ACCESIBILIDAD INDIVIDUAL COMO ELEMENTO DE EVALUACION DE LOS PLANES DE TRANSPORTE EN LA COMUNIDAD DE MADRID/ESPAÑA
}

\author{
(PERSONAL ACCESSIBILITY AS A FACTOR IN EVALUATION OF TRANSPORT PLANS \\ IN THE PROVINCE OF MADRID, SPAIN)
}

Andrés Monzón de Cáceres, Dr. Ingeniero de Caminos, Canales y Puertos

RESUMEN

La sociedad actual demanda una mayor capacidad de intercambio a todos los niveles. Para satisfacer esa demanda se necesitan más y mejores infraestructuras de transporte. Como las inversiones que exigen son muy elevadas, no pueden llevarse a cabo todas las propuestas. Hay, por tanto, que retrasar o desechar planes que resultan necesarios, e incluso urgentes para muchos, ante las limitaciones presupuestarias.

Surge así un problema de toma de decisiones. En el presente trabajo se propone un algoritmo de accesibilidad que permite cuantificar el grado de mejora que percibe el usuario al construir una nueva carretera. Se aplica a la evaluación de tres planes de transporte que han sido propuestos para resolver las difíciles condiciones de circulación del Area Metropolitana Madrileña. De este modo puede establecerse un método para optimizar el empleo de las dotaciones presupuestarias y, al mismo tiempo, evaluar los beneficios que se derivarán para el usuario.

Los resultados confirman las posibilidades del método. Se calculan los niveles de accesibilidad en el caso general (todos los motivos de viaje) y en el caso de los desplazamientos por motivos de trabajo. Los resultados permiten analizar tanto la mejora global de toda la provincia, como la de cada uno de sus munipios. Asimismo permite identificar los beneficios regionales.

\section{SUMMARY}

Society today presents a demand for greater interchange on all levels. To satisfy this demand we need more and better transport infrastructures. As this requires a high degree of investment, not all proposals can be carried out. Plans proven to be necesary and even urgent for some people have therefore to be postponed or rejected in the face of budgetary limitations.

This problem is solved by a decision-making process. This study proposes a accessibility indicator which allows us to quantify the degree of improvement benefitting the user when a new highway is built. The formula is applied to three transport plans which have been put forward as solutions to the difficult traffic conditions in the metropolitan area of Madrid. This enables us to establish a method for maximizing use of the available budget and at the same time to evaluate the user's benefit.

The results confirm the validity of the method. It's calculated the degree of general accessibility (for all motives for travelling) and the degree of accessibility for journeys to work. The results permit an analysis both of overall improvement in the whole province and in each municipality thereof. In this way we can determine regional benefits of each project.

\section{EL PAPEL DE LOS INDICADORES DE ACCESIBILIDAD EN LA ORDENACION URBANA $Y$ DEL TRANSPORTE}

Cada vez es más patente que la sociedad actual demande más posibilidades de interrelación, y para ello se necesita una red de transporte que vertebre el sistema territorial a todos los niveles: interurbano, intra e interregional, e internacional. Sin una adecuada red de transportes, íntimamente ligada a los planes de orde- nación del suelo, no es posible lograr un desarrollo armónico de las áreas metropolitanas, ni resolver las demandas de bienes y servicios de los habitantes de las zonas rurales.

Esta necesidad es reconocida cada año en los presupuestos de todos los estados, regiones y municipios, que destinan mayores contingentes monetarios para mejorar sus redes de transporte. Sin embargo, estas partidas son siempre limitadas y no cubren todas las 
necesidades. El "decisor político" necesita una herramienta que permita la toma de decisiones, teniendo en cuenta las mejoras que producen cada uno de los posibles planes alternativos de transporte. Esa herramienta de la planificación ha de permitir:

a) Identificar las zonas con mayor déficit viario y, en consecuencia, con menos posibilidades de movilidad.

b) Comparar planes alternativos que, alcanzando los objetivos de mejora global necesarios, tiendan a equilibrar las zonas, homogeneizándolas.

c) Evaluar el impacto y consecuencias de cada una de las alternativas.

Para cumplir estas funciones se vienen empleando en las últimas décadas los INDICADORES DE ACCESIBILIDAD, que han quedado ligados a los estudios y a la evaluación de los planes de transporte, con objeto de optimizar la toma de decisiones.

En términos generales, puede decirse que «la accesibilidad indica la facilidad con la cual un determinado uso del suelo puede ser alcanzado, desde una localización determinada, usando un sistema de transportes" (1).

La consideración de la accesibilidad ha servido para introducir una reorientación en los planes de transporte. Según Morris (2), «la planificación del transporte ha cambiado sustancialmente en un intento de integrarlo en la planificación de usos del suelo..., el punto central ha dejado de ser evitar la congestión, y ha pasado a ser proporcionar accesibilidad". Es decir, ha producido un cambio de signo en el planteamiento del problema. El objetivo ha pasado de ser negativo (evitar un mal: la congestión), para plantearse en términos positivos para el usuario: permitirle desarrollar con más libertad su capacidad de elección de destinos, esto es, mejorar su nivel de accesibilidad. Esta debe ser, dentro de las posibilidades de inversión y teniendo en cuenta los condicionantes de cada lugar, la finalidad última que debe presidir la toma de decisiones. Algunos investigadores (3) señalan que la ordenación del territorio debe basarse en la óptima conjugación de tres familias de criterios: accesibilidad, calidad del medio ambiente y capacidad de inversión. Las dos primeras se consideran contrapuestas, logrando su equilibrio a través del esfuerzo inversor.

La utilidad de los indicadores de accesibilidad en esta tarea se debe a su doble cualidad, como OBJETIVO a alcanzar y como METODOLOGIA DE PLANEAMIENTO de gran calidad y fácil utilización.

\section{MEDIDA DE LA SATISFACCION DEL USUARIO: ACCESIBILIDAD INDIVIDUAL}

Hay una enorme variedad de tipos de indicadores de accesibilidad; casi tanta como trabajos en los que se han empleado. Unos se centran únicamente en el estudio de la conectividad del grafo de la red, otros evalúan el potencial de generación de tráficos entre cada par de poblaciones, un tercer grupo desciende al análisis microeconómico intentando buscar la máxima utilidad del usuario, otros emplean métodos gráficos para medir el grado de movilidad, etc.

En todo viaje hay que considerar tres componentes: un ORIGEN, un DESTINO y el TRAYECTO que los separa. EI ORIGEN representa a los usuarios potenciales de la red de transportes; será más beneficioso realizar un determinado tramo de autopista o implantar una nueva línea de autobuses, cuanto mayor sea el número de posibles usuarios, es decir, cuanto mayor sea el valor de la componente que representa la influencia del origen.

En segundo lugar, para que se produzca un viaje ha de haber un DESTINO suficientemente atractivo como para superar la dificultad de recorrer la distancia que lo separa del origen. Evidentemente, esa "atracción" del destino dependerá de los bienes y servicios que el usuario puede alcanzar al término de su viaje. Por lo tanto, para un mismo destino, la "atracción" será diferente para los distintos motivos del viaje.

El tercer elemento es la consideración de la dificultad de superar la DISTANCIA que separa el origen y el destino. Su medida puede hacerse en términos de distancia física por el camino más corto, en tiempo o tiempo generalizado, en coste, etc. La dificultad está en que la medida de esa "impedancia" al desplazamiento entre el origen y el destino no depende exclusivamente de las características estructurales de la vía de transporte, sino que es cambiante con las diversas horas del dia y con la valoración que haga el usuario de las dificultades que pueda encontrar: congestión, peligrosidad, incomodidad del trazado, polución, etc.

Por tanto, todo indicador de accesibilidad ha de contar, para ser completo, con variables que representan los tres componentes del viaje.

En los últimos planes de carreteras de nuestro país también se han empezado a utilizar los indicadores de accesibilidad: en el Plan General de Carreteras 1984/91 del MOPU y en los de algunas Comunidades Autónomas como Madrid, Cataluña, Aragón, etc.

Sin embargo, los indicadores empleados corresponden, en general, a los de tipo Topológico, que consideran exclusivamente las distancias entre los nodos del grafo de la red. Por tanto, si bien dan una primera información sobre la accesibilidad, a escala regional no pro- 
porcionan un reflejo adecuado de la situación, pues no consideran la distinta importancia de los diversos origenes y destinos.

Entre los indicadores que toman en consideración los tres componentes del viaje se encuentran los MODELOS DE GRAVEDAD, desarrollados por analogía con la física de Newton, basándose en el concepto de interacción de Carrothers (5). Su formulación inicial corresponde a Hansen (6) que aplica una fórmula similar a la ley de la gravedad universal: las masas son las poblaciones y la distancia la separación entre las mismas, medida en tiempos medios.

Queremos proponer ahora un tipo de indicador, basado en los modelos de gravedad, pero que mida las posibilidades que proporciona al usuario individual una determinada infraestructura de transporte, para satisfacer su demanda de bienes y servicios.

Esa demanda puede satisfacerse en el propio nodo de origen, o en el resto de los nodos de la zona en estudio. En el primer caso, no se producirá un viaje, considerando como tales los de comunicación entre nodos. En el segundo, será necesario un desplazamiento, que tendrá características diferentes dependiendo de la localización relativa del nodo de origen y del de destino.

La situación de máxima accesibilidad sería aquella en que todos los usuarios pudiesen satisfacer sus necesidades en el lugar donde viven, por lo que no se necesitaria ningún desplazamiento. Por tanto, sólo se realizarian viajes por motivos de baja necesidad: culturales, turísticos, etc. El mínimo de accesibilidad se daría en aquellos casos en que para satisfacer la demanda de bienes y servicios se necesitase realizar una viaje largo.

La medida que se propone, para medir esa ACCESIBILIDAD INDIVIDUAL de cada nodo, tiene la siguiente formulación:

$$
A i=D i+\sum_{\forall j \neq i} \frac{D j}{f(d i j)}
$$

donde:

Aj es la ACCESIBILIDAD INDIVIDUAL del nodo i; Di es el número de bienes alcanzables en el origen; Dj es el número de bienes alcanzables en el nodo j; $f(d i j)$ mide la dificultad del desplazamiento de i a j.
Por tanto, la Accesibilidad Individual calcula el número de bienes alcanzables en el lugar donde se está situado, a los que suma los que se encuentran en todos los demás nodos, debidamente ponderados por la distancia que los separa. De este modo, pueden indentificarse aquellas zonas peor dotadas, más necesitadas de políticas tendentes a mejorar el equilibrio regional. Pueden diseñarse dos tipos de medidas: aumentar el número de bienes y servicios en las zonas más pobres o mejorar su comunicación con aquellas que puedan proporcionarles los bienes y servicios que demandan.

Se establece asi una medida del grado de SATISFACCION DEL USUARIO, asociado a su lugar de residencia. Pueden medirse, también, lós incrementos que producirán políticas alternativas que tiendan a mejorar la situación.

Estos estudios pueden considerar todas las necesidades del usuario - todos los motivos del viaje - o corresponder a trabajos sectoriales que seleccionen unos determinados motivos de viaje. En cada caso habrá que elegir las variables maś adecuadas.

En estas páginas se estudiará, en el ámbito de la Comunidad de Madrid, el caso general (todos los motivos de viaje) y un caso sectorial (los desplazamientos al trabajo).

Las variables que más se emplearán son las siguientes:

GENERAL: $\mathrm{Di}=\mathrm{Pi}$, población del nodo i; $\mathrm{Dj}=\mathrm{Pj}$, población del nodo $\mathrm{j}$.

EMPLEO: $\mathrm{Di}=\mathrm{Ei}$, puestos de trabajo en el nodo $\mathrm{i}$; $D j=E j$, puestos de trabajo en el nodo $\mathrm{j}$.

Como función de la distancia se tomará una de tipo exponencial que, según plantea Koening (7), se ajusta más adecuadamente a la maximización de la utilidad del usuario. Es la siguiente:

$$
f(d i j)=e^{D i j}
$$

donde Dij es la DISTANCIA OPERACIONAL (8), que se basa en la medida de la distancia mínima entre el origen y el destino y en la consideración de las condiciones estructurales y de circulación bajo un Coeficiente de Impedancia para cada tramo. La formulación de esa distancia se recoge en la tabla 1: la consideración del Coeficiente de Impedancia puede, en el caso más desfavorable, llegar a duplicar la distancia real entre los nodos.

Para facilitar el cálculo, la medida de la distancia se hará en decenas de kilómetros. 
VALORES DEL COEFICIENTE DE IMPEDANCIA Y CALCULO DE LA DISTANCIA OPERACIONAL

CATEgORIA A. Características de la Plataforma

Anchura de la Plataforma: $<6 \mathrm{~m}$

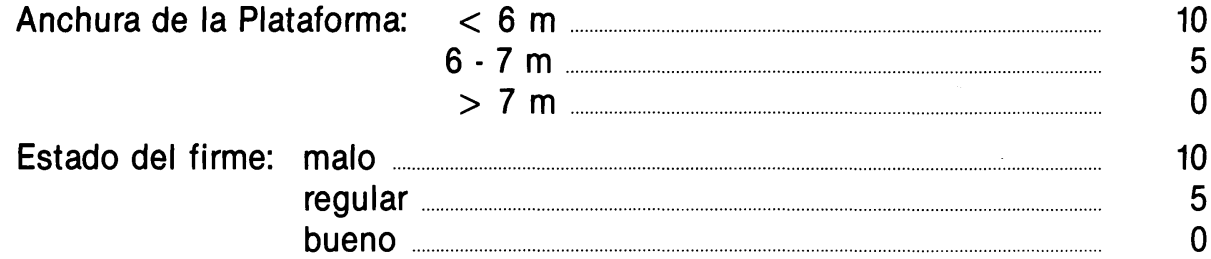

CATEGORIA B. Características del tráfico

IMD: > 40.000 vehículos/día

15

20 - 40.000 vehículos/dia

10

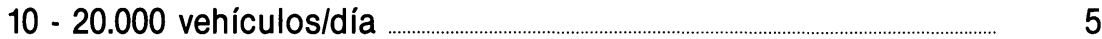

$5 \cdot 10.000$ vehículos/dia .........._._._._._._.

$<5.000$ vehículos/día ....._________._. 0

Vehículos Pesados: > 5.000 vehículos/día ...._._. 10

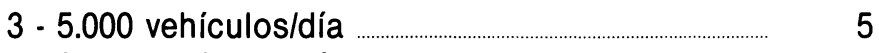

$1 \cdot 3.000$ vehículos/día ......................................... 3

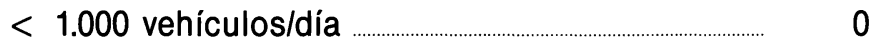

CATEGORIA C. Condiciones de circulación

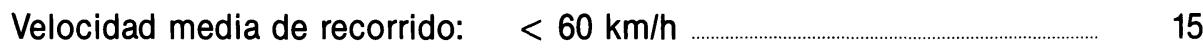

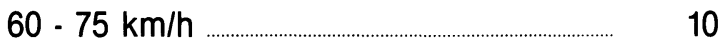

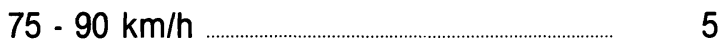

$>90 \mathrm{~km} / \mathrm{h} \ldots$

Nivel de Servicio: E ...

$D$

A, B ó C _ _ _ n

Travesías Congestionadas: 1er. grado ...._._.

$2 .^{\circ}$ grado ............ 5

Travesías con problemas viarios: De primer grado ............................. 10

Peligrosidad relativa de la red: $\quad>30$ accidentes con víctimas al año $\quad 10$

10 - 30 accidentes con víctimas al año 5

$<10$ accidentes con víctimas al año 0

COEFICIENTE DE IMPEDANCIA $=\mathrm{lij}^{1}=\mathrm{A}^{1} \mathrm{ij}+\mathrm{A}^{2} \mathrm{ij}+\mathrm{B}^{1} \mathrm{ij}+\mathrm{B}^{2} \mathrm{ij}+\mathrm{C}^{1} \mathrm{ij}+\ldots+\mathrm{C}^{5} \mathrm{ij}$

DISTANCIA OPERACIONAL $=\mathrm{Dij}=\operatorname{dij}(1+\mathrm{lij} / 100)$

siendo dij la distancia física del nodo $\mathrm{i}$ al $\mathrm{j}$

$\mathrm{dij}<\mathrm{Dij}<2 \mathrm{dij}$ 


\section{LA RED DE CARRETERAS DE LA PROVINCIA DE MADRID. CALCULO DE LA ACCESIBILIDAD INDIVIDUAL EN LA SITUACION ACTUAL}

No es preciso justificar la necesidad de mejorar la red de trasportes de la provincia de Madrid; las críticas sobre sus deficiencias -especialmente dentro del Area Metropolitana - son noticia frecuente en la prensa diaria y revistas especializadas.

Esta Comunidad uniprovincial tiene una infraestructura de transportes en la que coinciden tráficos de tipos diversos, al no contar con una red suficientemente jerarquizada. La disposición radial de las grandes vías de penetración, convergentes en el centro, y la falta de itinerarios transversales adecuados hace que se mezclen los tráficos de largo recorrido - buena parte de paso hacia otras regiones - con los tráficos provinciales y los propios del Area Metropolitana.

El presente estudio se ciñe a la red de carreteras. En primer lugar, se establece un GRAFO SIMPLIFICADO partiendo de los municipios de más de 5.000 habitantes, los extremos provinciales y las intersecciones de las carreteras que los unen. Resultan así 70 nodos, que suman el $97 \%$ de la población de la provincia. Los tramos de carreteras considerados incluyen la totalidad de la Red del Estado, de la Básica de Primer Orden, gran parte de la de Segundo Orden y algunos tramos de la Local Estructurante.

En este grafo están incluidos todos los tramos con una IMD (Intensidad Media Diaria) superior a los 5.000 veh./dia y con una intensidad de vehículos pesados superior a los 1.000 diarios. Por tanto, si bien no es completo, resulta suficientemente representativo de la red provincial, tanto de la que es competencia del MOPU como de la que ha pasado a ser administrada por la Comunidad de Madrid.

En la Figura 1, se detallan los tramos considerados en el Grafo Simplificado y la denominación de los nodos que corresponden a poblaciones. Los restantes son intersecciones de carreteras que es necesario incluir para considerar todos los tramos significativos y dar conexión al grafo.

Para considerar la influencia exterior se han determinado unas poblaciones ficticias basándose en los tráficos de entrada en los extremos provinciales (9). También se asigna a esos nodos un número de puestos de trabajo de acuerdo con el número de residentes en la provincia de Madrid que se desplazan al trabajo a las colindantes (10). Los datos correspondientes a cada nodo se recogen en el Anexo I.
Sobre este grafo se ha realizado el cálculo de la ACCESIBILIDAD INDIVIDUAL en los dos casos definidos en el apartado anterior: viajes por todos los motivos (General) y viajes al trabajo (Empleo).

\section{Accesibilidad Individual General}

La Accesibilidad Individual General del nodo i vendrá definida por el siguiente cálculo:

$$
A G i=P i+\sum_{\forall j \neq i} \frac{P j}{e^{0,1 d i j}}
$$

Los resultados para cada uno de los nodos vienen recogidos en la Tabla 2. Se dan los resultados por el orden numérico de los nodos y por el valor de la accesibilidad de cada uno.

Estos valores de la accesibilidad corresponden a la situación de la red viaria antes del Plan de Carreteras de 1985 de la Comunidad de Madrid (11), del que se han llevado a la práctica sólo algunas partes, y otras han sufrido modificaciones sustanciales.

Del estudio de la Tabla 2 cabe destacar la posición privilegiada de los habitantes de la capital. La razón es clara: la mayor parte de los bienes y servicios los pueden alcanzar sin salir de la ciudad. Su nivel de accesibilidad es 2,5 veces superior al del nodo siguiente, 3 veces el del tercero (Alcorcón) y casi 12 veces el valor medio de los 70 nodos considerados.

El segundo valor más alto es el del nodo 58, que es una intersección de carreteras. En principio sorprende este resultado pues, al no tener población, la contribución del primer sumando es nula. La razón hay que buscarla en su proximidad a Madrid y a otras poblaciones importantes (Alcorcón, Móstoles, Leganés...). En la misma situación se encuentra Aravaca (nodo 60) con un valor de accesibilidad individual muy superior al que le correspondería por su población, y el nodo 56 en la $\mathrm{N}$-IV. El resto de los nodos con valores más destacados son los situados en el suroeste del Area Metropolitana.

A continuación se encuentran los habitantes del resto de los municipios del Area Metropolitana y de las zonas próximas a los grandes ejes radiales.

Los niveles más bajos corresponden a los núcleos periféricos.

En la Figura 2 pueden comprobarse, de modo gráfico, los niveles de accesibilidad comentados. 


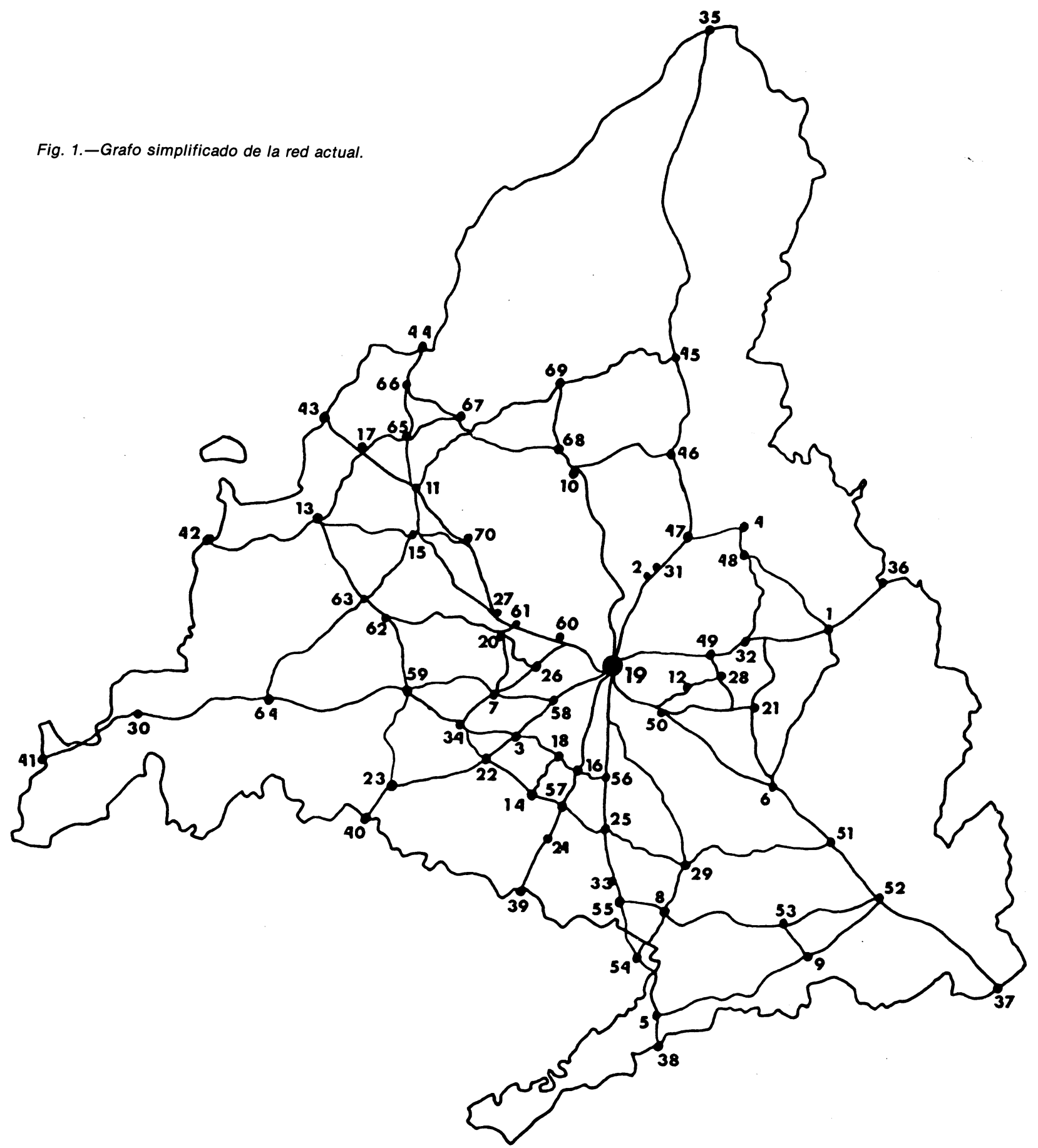

1. Alcalá de Henares

2. Alcobendas

3. Alcorcón

4. Algete

5. Aranjuez

6. Arganda

7. Boadilla del Monte

8. Ciempozuelos

9. Colmenar de Oreja

10. Colmenar Viejo

11. Collado Villalba

12. Coslada

13. Los Escoriales

14. Fuenlabrada

15. Galapagar

16. Getafe
17. Guadarrama

18. Leganés

19. Madrid

20. Majadahonda

21. Mejorada del Campo

22. Móstoles

23. Navalcarnero

24. Parla

25. Pinto

26. Pozuelo de Alarcón

27. Las Rozas de Madrid

28. San Fernando de Henares

29. San Martín de la Vega

30. San Martín de Valdeiglesias

31. San Sebastián de los Reyes

32. Torrejón de Ardoz
33. Valdemoro

34. Villaviciosa de Odón

45. Venturada

46. San Agustín de Guadalix

48. Cobeña

51. Perales de Tajuña

52. Villarejo de Salvanés

59. Brunete

60. Aravaca

61. El Plantío

63. Valdemorillo

64. Chapinería

65. Collado Mediano

67. Cerceda-El Boalo

69. Soto del Real

70. Torrelodones 
TABLA 2

ACCESIBILIDAD INDIVIDUAL GENERAL EN LA SITUACION ACTUAL

\begin{tabular}{|c|c|c|c|c|c|}
\hline Nodo & $A^{G}$ & Nodo & $A^{G}$ & Nodo & $A^{G}$ \\
\hline $\begin{array}{l}30 \\
9 \\
53 \\
5 i \\
69 \\
64 \\
45 \\
42 \\
51 \\
37 \\
66 \\
4 ! \\
67 \\
65 \\
44 \\
35 \\
17 \\
43 \\
13 \\
11 \\
68 \\
63 \\
46\end{array}$ & $\begin{array}{l}16895.6516077 \\
19108.8826512 \\
19371.0569918 \\
21815.3514353 \\
23658.598231 \\
26897.5827483 \\
29459.0203194 \\
31169.24643666 \\
35331.67066 ? \\
39899.9927024 \\
39146.4847851 \\
40457.9885429 \\
41122.7671823 \\
43797.3932237 \\
43919.38333287 \\
45419.3863612 \\
46589.8481416 \\
48949.5074119 \\
53366.5114304 \\
59949.5820385 \\
6 j 381.3354114 \\
67244.6695675 \\
77561.142948\end{array}$ & $\begin{array}{l}15 \\
54 \\
10 \\
8 \\
48 \\
70 \\
5 \\
02 \\
38 \\
4 \\
40 \\
6 \\
29 \\
23 \\
36 \\
55 \\
39 \\
33 \\
21 \\
59 \\
47 \\
1 \\
32 \\
24\end{array}$ & 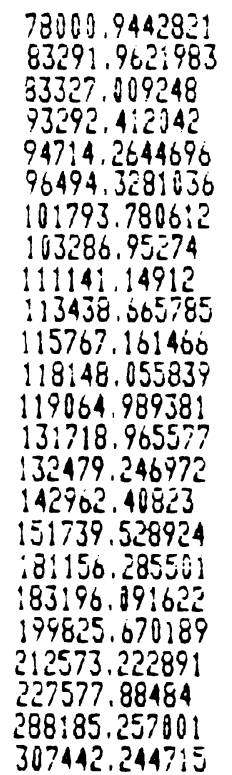 & $\begin{array}{l}27 \\
20 \\
31 \\
12 \\
25 \\
2 \\
20 \\
28 \\
34 \\
61 \\
14 \\
49 \\
57 \\
7 \\
22 \\
56 \\
18 \\
60 \\
16 \\
50 \\
3 \\
58 \\
19\end{array}$ & $\begin{array}{l}320463.834689 \\
332042.033127 \\
329427.543371 \\
334: 27 \\
347104.287503 \\
356148.676286 \\
357444.520568 \\
358764.800335 \\
373584.882564 \\
408763.612249 \\
417022.742022 \\
424408.439815 \\
427335.815348 \\
454170.9714 \\
503426.294958 \\
535274.960226 \\
556300.33124 \\
617836.216649 \\
690998.257064 \\
722257.61303 \\
745755.23644 \\
845542.63253 \\
1.29942026468 E+6 \\
3.17291562225 E+6\end{array}$ \\
\hline
\end{tabular}

TABLA 3

ACCESIBILIDAD INDIVIDUAL AL EMPLEO EN LA SITUACION ACTUAL

\begin{tabular}{|c|c|}
\hline Nodo & $A^{E}$ \\
\hline $\begin{array}{l}35 \\
4 i \\
3 i \\
4 i \\
30 \\
44 \\
43 \\
9 \\
53 \\
50 \\
50 \\
64 \\
09 \\
1 i \\
55 \\
45 \\
35 \\
6 i \\
5 \vdots \\
40 \\
10 \\
15 \\
5\end{array}$ & 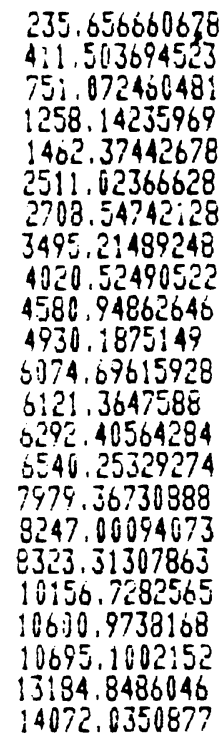 \\
\hline
\end{tabular}

\begin{tabular}{|c|c|}
\hline Nodo & $A^{E}$ \\
\hline $\begin{array}{l}3 i \\
65 \\
54 \\
58 \\
34 \\
15 \\
25 \\
8 \\
45 \\
65 \\
10 \\
48 \\
70 \\
4 \\
29 \\
55 \\
6 \\
33 \\
59 \\
\vdots \\
21 \\
24 \\
47 \\
14\end{array}$ & $\begin{array}{l}14259.6438081 \\
14776.4688574 \\
16062.59125 \\
16475.1180487 \\
18580.9251301 \\
20209.8080020 \\
21070.0980905 \\
21258.3990533 \\
21362.0034101 \\
23351.838787 \\
23625.4206705 \\
23859.9018211 \\
27251.9046844 \\
30174.5043726 \\
31359.8811583 \\
33710.653907 \\
38831.9532008 \\
43665.9016524 \\
46302.6819082 \\
46544.9664547 \\
54944.0008359 \\
58276.4033901 \\
59727.7946642 \\
79617.4594023\end{array}$ \\
\hline
\end{tabular}

\begin{tabular}{|c|c|}
\hline Nodo & $A^{E}$ \\
\hline $\begin{array}{l}32 \\
25 \\
34 \\
31 \\
27 \\
20 \\
57 \\
2 \\
26 \\
12 \\
22 \\
28 \\
18 \\
61 \\
49 \\
7 \\
50 \\
16 \\
3 \\
60 \\
50 \\
58 \\
19\end{array}$ & $\begin{array}{l}85710.6155414 \\
86895.6070768 \\
94399.70159166 \\
94913.9367899 \\
96053.1298833 \\
96593.4157285 \\
99156.2624542 \\
103113.5402 \\
103391.959443 \\
103399.006853 \\
112422.699808 \\
114776.090632 \\
133047.343256 \\
133739.963886 \\
135002.472362 \\
150943.345642 \\
159951.624445 \\
184089.827524 \\
2320.6 .680217 \\
272390.260634 \\
254929.14171 \\
424286.276613 \\
1.10919673772 \mathrm{E}+6\end{array}$ \\
\hline
\end{tabular}




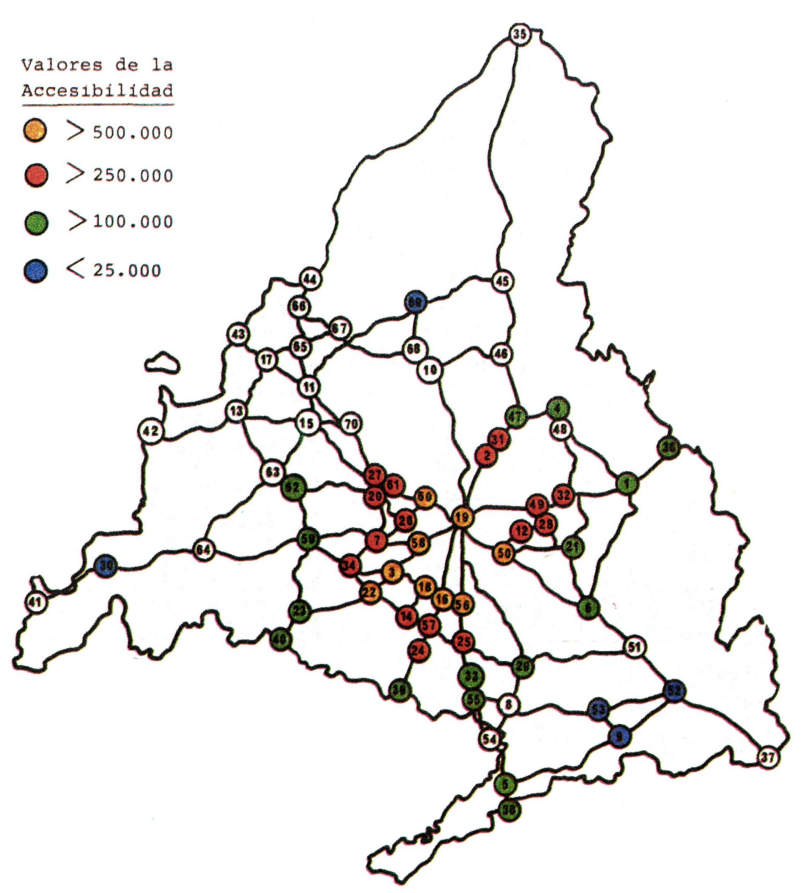

Fig. 2. - Niveles de Accesibilidad Individual General en la Situación Actual.

Destaca la posición privilegiada de los municipios del Area Metropolitana, que se prolonga por los grandes ejes radiales. Las zonas con menores oportunidades son la del norte (Sierra) y los extremos sureste y suroeste de la provincia.

\section{Accesibilidad individual al Empleo}

Analicemos ahora la Accesibilidad Individual al Empleo. En este caso, la formulación de la accesibilidad del nodo i será la siguiente:

$$
A E_{i}=E i+\sum_{\forall j \neq i} \frac{E j}{e^{0,1 d i j}}
$$

Los resultados de este cáculo sectorial son los que se recogen en la Tabla 3.

En este caso, el mayor valor de la accesibilidad lo da, también, Madrid, aun con mayor diferencia respecto al resto: 2,6 veces la del nodo $58,4,4$ veces la del nodo 50 - que es el siguiente en este caso-, y 15 veces el nivel medio.

La causa de estos resultados es la concentración de puestos de trabajo que tiene el municipio de Madrid, y especialmente la Almendra Central (cfr. Anexo II).

Mejoran su posición respecto al caso General los municipios situados en las proximidades de los ejes radiales de la $\mathrm{N}-\mathrm{Il}$ y $\mathrm{N}-\mathrm{VI}$, donde ha habido un proceso

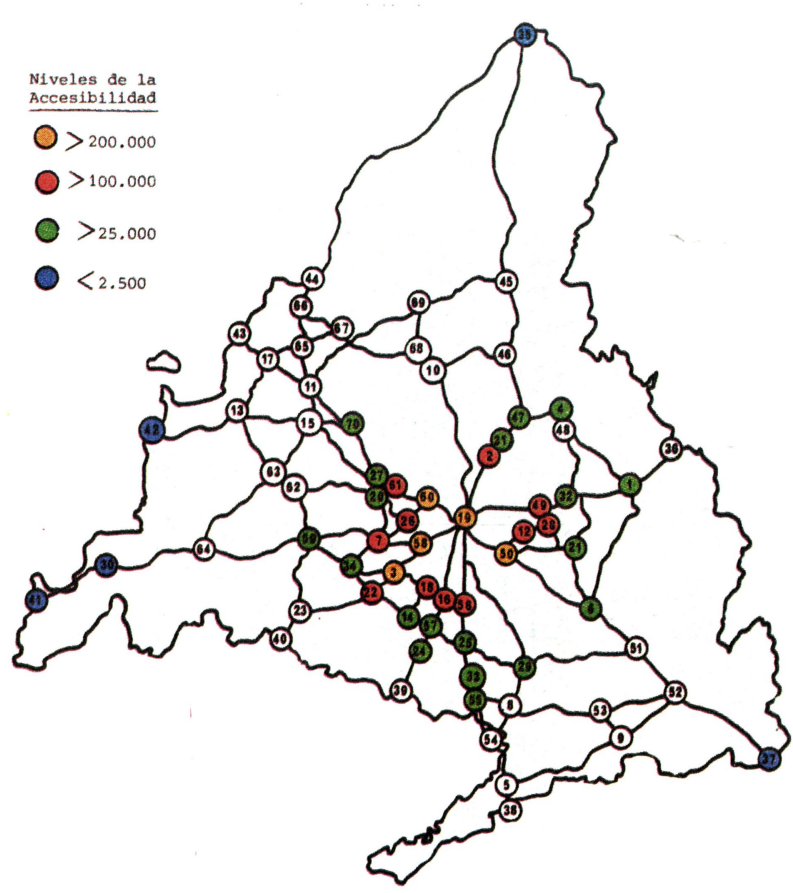

Fig. 3. - Niveles de Accesibilidad individual al Empleo en la Situación Actual.

de localización industrial y del sector servicios. Es el caso del nodo 50, Aravaca (nodo 60), Boadilla del Monte (nodo 7), El Plantío (nodo 71), nodo 49, etc.

Por el contrario, el déficit de puestos de trabajo de la zona sur explica el retroceso de posiciones de los municipios ahí situados: Alcorcón (nodo 3), Getafe (nodo 16 ), Leganés (nodo 18), Móstoles (nodo 22), etc.

En cualquier caso se mantiene, con las ligeras modificaciones, la misma situación del caso general: Ia zona con más accesibilidad es la central y las regiones más desfavorecidas las periféricas.

En la Figura 3 se tiene una imagen de los Niveles de Accesibilidad Individual al Empleo.

Una vez calculados los Niveles de Accesibilidad Individual en la situación actual, pasemos a analizar qué mejoras introducirían algunos planes de transporte que podrian llevarse a cabo.

\section{EVALUACION DE TRES PLANES DE TRANSPORTE PROPUESTOS EN LA COMUNIDAD DE MADRID}

Con la metodología propuesta se estudian tres planes de transporte propuestos por diversos grupos sociales y entidades oficiales en el ámbito de la Comunidad de Madrid. En cada uno de los casos seguiremos el proceso siguiente: 
- Modificación del grafo de la red de carreteras y cálculo de las nuevas distancias entre nodos.

- Cálculo de la Accesibilidad individual en el caso General y en el sectorial del Empleo.

- Análisis de las mejoras sobre la situación actual.

Los casos considerados son los siguientes:

\section{A. Cinturón de Circunvalación}

Desde los años 70 se empezó a considerar la posibilidad de construir un anillo de circunvalación exterior a la M-30 que, actualmente, es una vía de carácter urbano. Sin embargo, las grandes inversiones necesarias y la distribución de competencias entre las administraciones central y autonómica han supuesto un retraso en la solución de los problemas del transporte en el ámbito provincial. No obstante, este proyecto no fue contemplado ni en el Plan General de Carreteras del
MOPU (12), ni en el Plan de Carreteras de la Comunidad de Madrid (11). Sin embargo, en los programas de casi todos los partidos políticos para las elecciones locales y autonómicas de 1987 se planteaba la necesidad de una infraestructura de este tipo. En el presente año se ha elaborado un Plan Estratégico (13) para la provincia que prevé la construcción de una serie de corredores que, en su conjunto, formarán otra vía de circunvalación, aunque las características no son total. mente homogéneas.

Queda abierta, también, la posibilidad futura de construir un anillo exterior al Area Metropolitana con características de autovía. Se realiza el estudio de una vía de este tipo, según el trazado que se propone en la Figura 4. Dicho trazado intenta aprovechar al máximo los viales existentes, evitar zonas urbanas de alta densidad donde sería difícil la reserva de suelo necesaria, y minimizar el impacto ambiental.

TABLA 4

ACCESIBILIDAD INDIVIDUAL GENERAL CON EL CINTURON DE CIRCUNVALACION Y COMPARACION CON LA SITUACION ACTUAL

\begin{tabular}{|c|c|c|}
\hline Nodo & $\begin{array}{l}\text { Accesibilidad } \\
\text { Sit. Inicial }\end{array}$ & $\begin{array}{l}\text { Accesibilidad } \\
\text { con Cinturón }\end{array}$ \\
\hline $\begin{array}{l}1 \\
2 \\
3 \\
4 \\
5 \\
6 \\
7 \\
8 \\
9 \\
10 \\
11 \\
12 \\
13 \\
14 \\
15 \\
16 \\
17 \\
18 \\
19 \\
20 \\
21 \\
22 \\
23 \\
24 \\
25 \\
26 \\
27 \\
28 \\
29 \\
30 \\
31 \\
32 \\
33 \\
34 \\
35 \\
36 \\
37\end{array}$ & 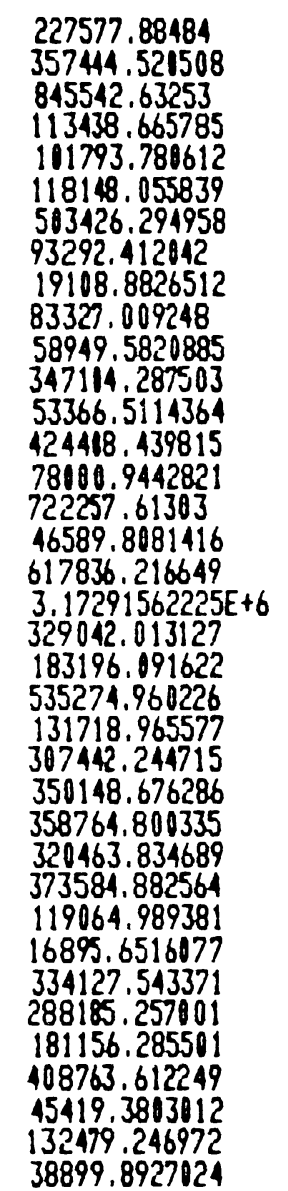 & 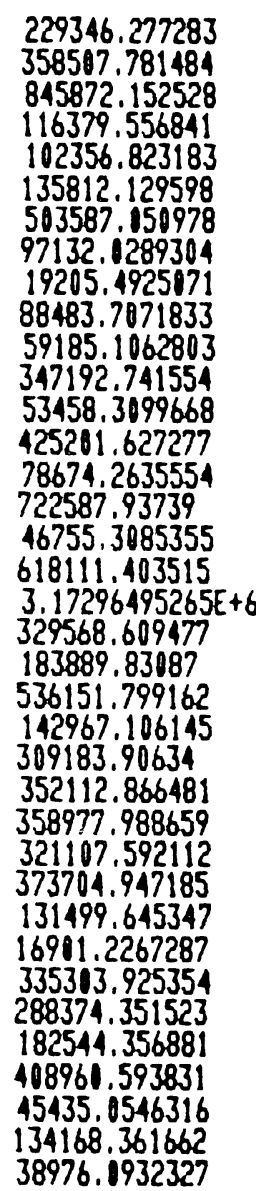 \\
\hline
\end{tabular}

\begin{tabular}{|c|c|c|}
\hline Nodo & $\begin{array}{c}\text { Accesibilidad } \\
\text { Sit. Inicial }\end{array}$ & $\begin{array}{l}\text { Accesibilidad } \\
\text { con Cinturón }\end{array}$ \\
\hline $\begin{array}{l}38 \\
39 \\
40 \\
41 \\
42 \\
43 \\
44 \\
45 \\
46 \\
17 \\
48 \\
19 \\
50 \\
51 \\
52 \\
53 \\
54 \\
55 \\
56 \\
57 \\
58 \\
59 \\
60 \\
61 \\
62 \\
63 \\
64 \\
65 \\
66 \\
67 \\
68 \\
69 \\
70 \\
71 \\
72 \\
73\end{array}$ & 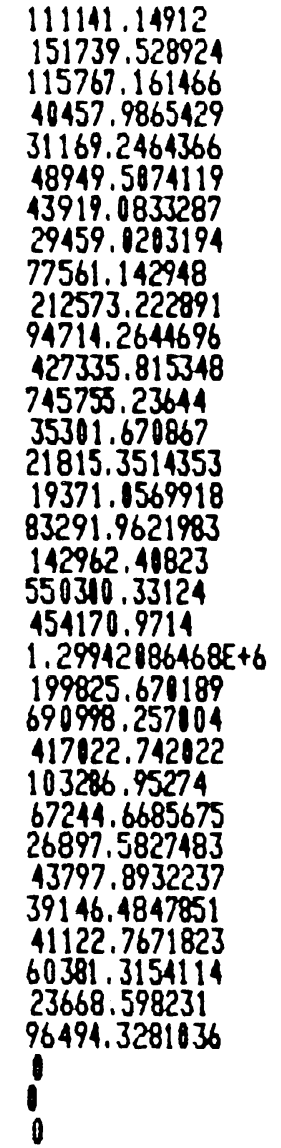 & 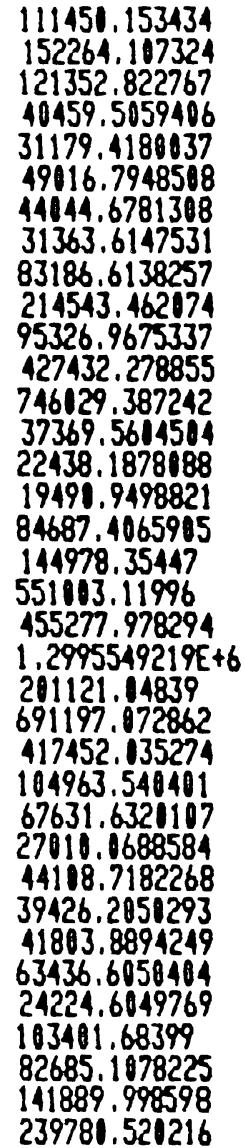 \\
\hline
\end{tabular}




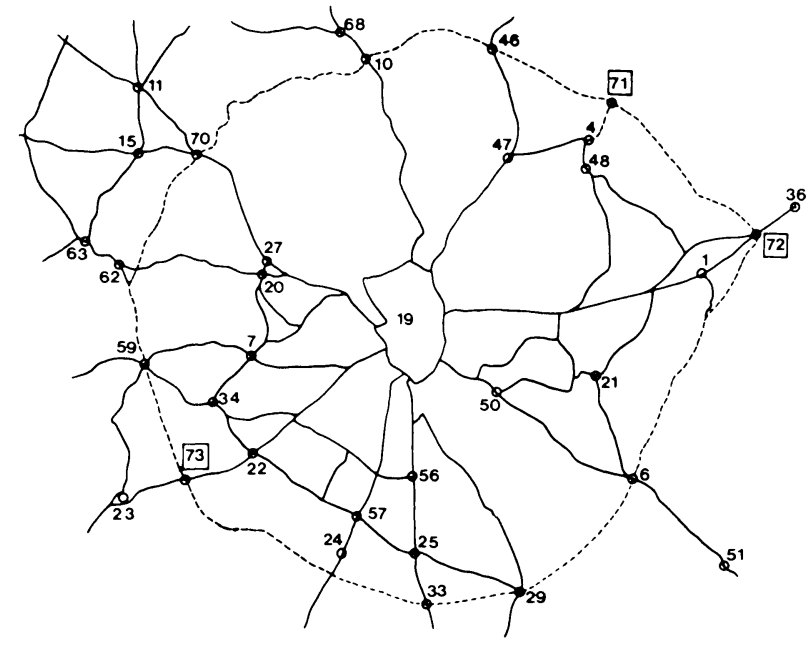

Fig. 4.-Trazado Del Cinturón de Circunvalación.
Se ha determinado la nueva matriz de distancias, contando con el Cinturón y se ha realizado el cálculo de la accesibilidad en los dos supuestos del estudio. Los resultados vienen recogidos en las Tablas 4 y 5 . En ellas se detallan los nuevos valores de la Accesibilidad Individual y se comparan con la situación actual.

En el caso General, los nodos que tienen mayores in. crementos de Accesibilidad son los situados sobre el nuevo Cinturón y sus proximidades. Con mejoras superiores al $100 \%$ se encuentran Arganda (nodo 6) y San Martín de la Vega (nodo 29). Con incrementos superiores al $50 \%$ están Navalcarnero (nodo 23), San Agustín de Guadalix (nodo 46), Torrelodones (nodo 70), Venturada (nodo 45), Colmenar Viejo (nodo 10), etc. Las

TABLA 5

ACCESIBILIDAD INDIVIDUAL AL EMPLEO CON EL CINTURON DE CIRCUNVALACION Y COMPARACION CON LA SITUACION ACTUAL

\begin{tabular}{|c|c|c|}
\hline Nodo & $\begin{array}{c}\text { Accesibilidad } \\
\text { Sit. Inicial }\end{array}$ & $\begin{array}{l}\text { Accesibilidad } \\
\text { con Cinturón }\end{array}$ \\
\hline $\begin{array}{l}1 \\
2 \\
3 \\
4 \\
5 \\
6 \\
7 \\
8 \\
9 \\
10 \\
11 \\
12 \\
13 \\
14 \\
15 \\
16 \\
17 \\
18 \\
19 \\
20 \\
21 \\
22 \\
23 \\
24 \\
25 \\
26 \\
27 \\
28 \\
29 \\
30 \\
31 \\
32 \\
33 \\
34 \\
35 \\
36 \\
37\end{array}$ & 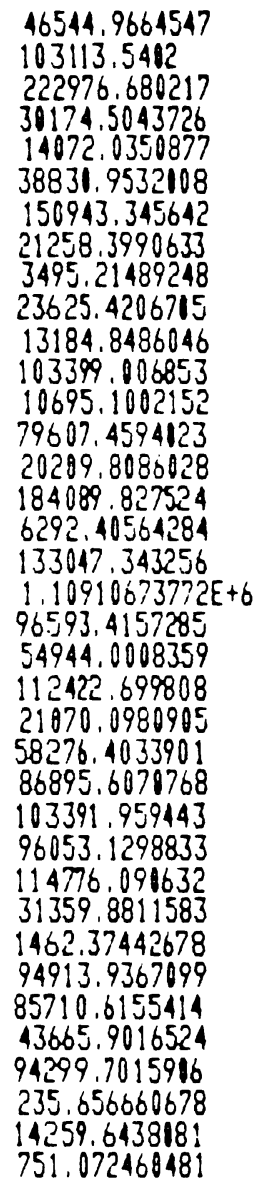 & 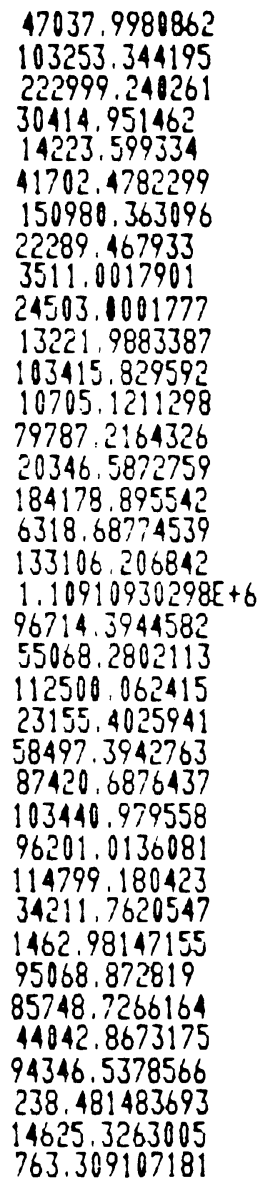 \\
\hline
\end{tabular}

\begin{tabular}{|c|c|c|}
\hline Nodo & $\begin{array}{c}\text { Accesibilidad } \\
\text { Sit. Inicial }\end{array}$ & $\begin{array}{l}\text { Accesibilidad } \\
\text { con Cinturón }\end{array}$ \\
\hline $\begin{array}{l}38 \\
39 \\
40 \\
41 \\
12 \\
43 \\
14 \\
45 \\
46 \\
47 \\
48 \\
49 \\
50 \\
51 \\
52 \\
53 \\
54 \\
55 \\
56 \\
57 \\
58 \\
59 \\
60 \\
61 \\
62 \\
63 \\
64 \\
65 \\
66 \\
67 \\
68 \\
69 \\
70 \\
71 \\
72 \\
73\end{array}$ & $\begin{array}{l}8247.00094173 \\
18680.0251301 \\
10601.9738168 \\
411.503694523 \\
1258.14235969 \\
2708.54742128 \\
25111.02366628 \\
7979.36730888 \\
21362.0034101 \\
59728.7946642 \\
23859.9018211 \\
135813.472362 \\
254930.14171 \\
10156.7282565 \\
4580.94862646 \\
1020.52490522 \\
16063.59125 \\
33711.053907 \\
159952.624445 \\
99157.2624542 \\
424287.276613 \\
46302.6819882 \\
232280.260634 \\
133739.963886 \\
23252.838787 \\
14776.4088574 \\
6074.69615928 \\
6540.253229274 \\
4931.1875149 \\
8323.31307863 \\
16476.1186487 \\
6121.36477888 \\
27251.9046844 \\
0 \\
1 \\
0\end{array}$ & $\begin{array}{l}8330.18116269 \\
18746.5863059 \\
11636.5053872 \\
111.669133523 \\
1259.25270868 \\
2719.23292677 \\
2531.92360126 \\
8322.61273289 \\
22357.1172807 \\
59994.7729695 \\
24005.6014843 \\
135021.059597 \\
254979.247615 \\
10488.4961401 \\
4680.87516258 \\
4039.83324897 \\
16436.3791416 \\
34248.8140141 \\
160145.765196 \\
99411.0811725 \\
424294.212977 \\
46488.8144508 \\
232325.983858 \\
133838.606797 \\
23586.6480621 \\
14837.1129767 \\
6086.88897946 \\
6591.72875968 \\
4977.70111756 \\
8437.25249028 \\
16989.6643375 \\
6211.76654287 \\
28359.0351821 \\
20787.9412645 \\
22339.3213333 \\
47102.2143122\end{array}$ \\
\hline
\end{tabular}




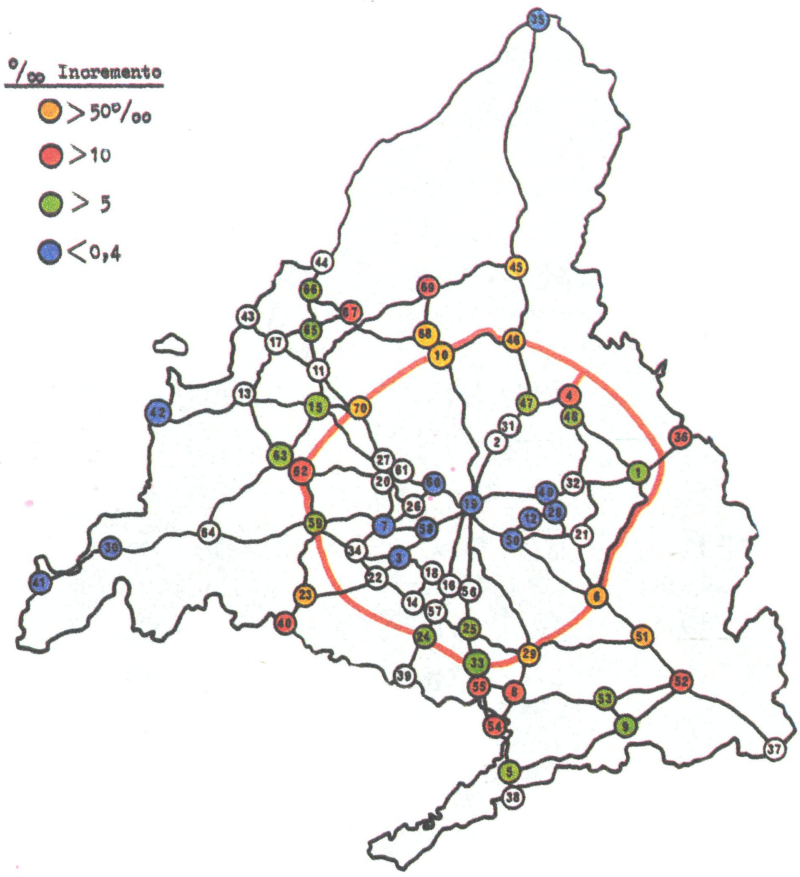

Fig. 5. - Incremento de Accesibilidad Individual General con el Cinturón de Circunvalación.

zonas menos afectada son, por un lado, las más internas del Area Metropolitana y, por otro, las más periféricas. Estos resultados pueden analizarse en la Figura 5 . Los incrementos que se producen, si bien son significativos en las zonas indicadas, no dan un cambio sustancial a los niveles de accesibilidad de la provincia, por los fuertes contrastes que se han detallado en el apartado anterior. Sin embargo, el Cinturón de Circunvalación contribuiría al equilibrio regional, pues los mayores incrementos se producen precisamente en puntos con niveles bajos o medios de accesibilidad en la situación actual. Esto quiere decir que permitiría suavizar el aislamiento de estas zonas.

En el caso de los desplazamientos al trabajo, los incrementos de accesibilidad que introduce el Cinturón son ligeramente inferiores al caso General. Los nodos más beneficiados están sobre la N-V: Navalcarnero (nodo 23) y el nodo 40. A continuación están San Martín de la Vega (nodo 29), Arganda (nodo 6), Ciempozuelos (nodo 8), etc. Los menos afectados están localizados, también, en el centro de la provincia y en la periferia. Por tanto, los efectos sobre los viajes al trabajo son similares -cfr. Figura 6- al caso General, donde se consideran todos los motivos de viaje posibles.

\section{B. Distribuidor Sur}

Algunos de los sectores de la oposición plantearon, a nivel municipal, la construcción de un eje - con carac-

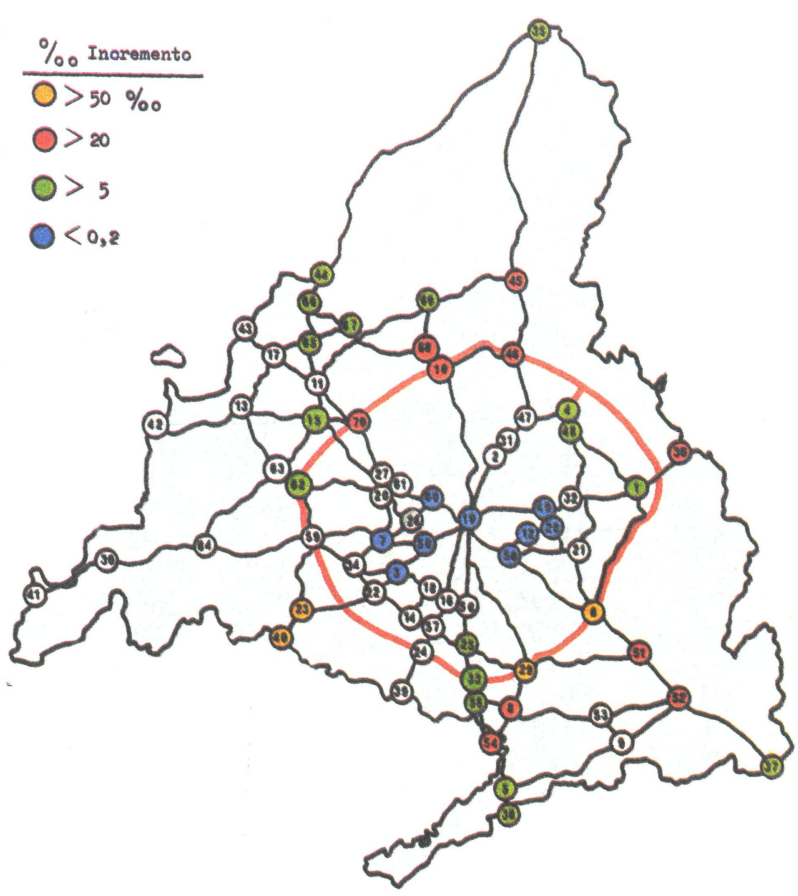

Fig. 6. - Incremento de Accesibilidad Individual al Empleo con el Cinturón de Circunvalación.

terísticas de autopista- que enlazase la N-II con la $\mathrm{N}$ V. En el Plan Estratégico de la Región (13), presentado recientemente por la Comunidad de Madrid, se incluye el Distribuidor Sur, aunque no tiene el carácter de autopista y su trazado es más próximo al centro del Area Metropolitana. El trazado propuesto por los grupos de la oposición fue el que se recoge en la Figura 7.

Teniendo en cuenta las modificaciones que esta nueva vía introduciría en el grafo de la red, se determinan las distancias entre cada par de nodos y se hace el cálculo de la Accesibilidad Individual en los dos supuestos que se vienen considerando: General y al Empleo. Los resultados se detallan en las Tablas 6 y 7 .

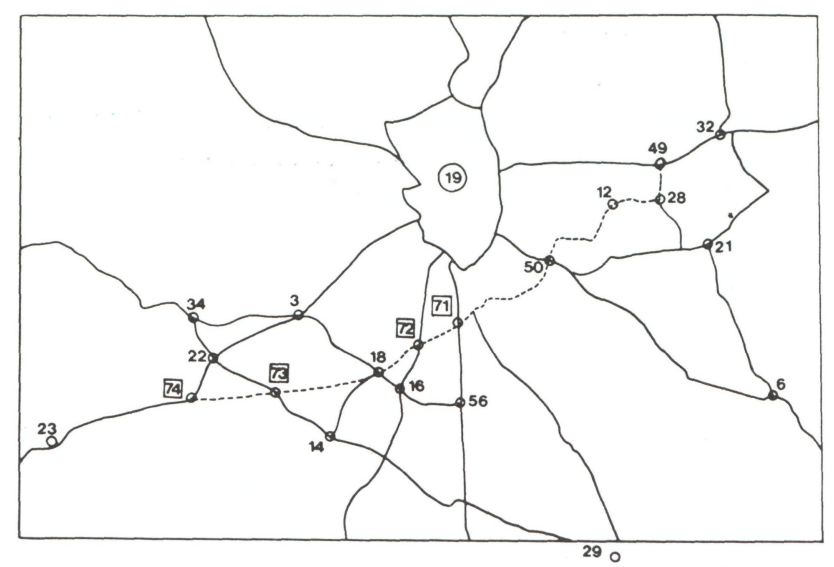

Fig. 7.-Trazado del Distribuidor Sur. 
TABLA 6

ACCESIBILIDAD INDIVIDUAL GENERAL CON EL DISTRIBUIDOR SUR. INCREMENTOS RESPECTO A LA SITUACION INICIAL

\begin{tabular}{|c|c|c|}
\hline Nodo & $\begin{array}{l}\text { Accesibilidad } \\
\text { Sit. Inicial }\end{array}$ & $\begin{array}{l}\text { Accesibilidad con } \\
\text { Distribuidor Sur }\end{array}$ \\
\hline $\begin{array}{l}1 \\
2 \\
3 \\
1 \\
5 \\
6 \\
7 \\
8 \\
9 \\
10 \\
11 \\
12 \\
13 \\
14 \\
15 \\
16 \\
17 \\
18 \\
19 \\
20 \\
21 \\
22 \\
23 \\
24 \\
25 \\
26 \\
27 \\
28 \\
29 \\
30 \\
31 \\
32 \\
33 \\
34 \\
35 \\
36 \\
37\end{array}$ & 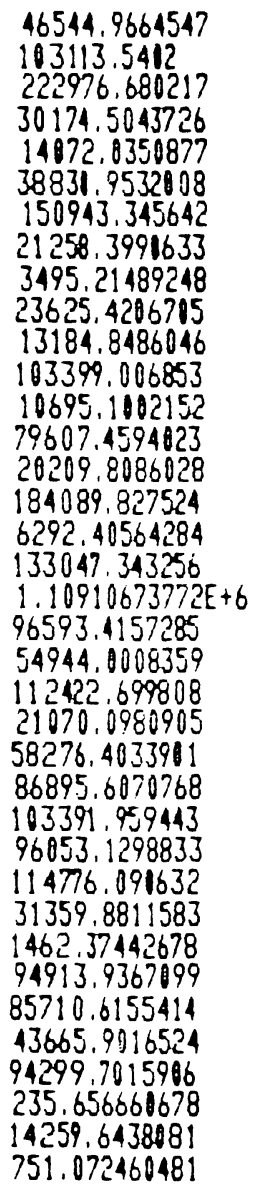 & 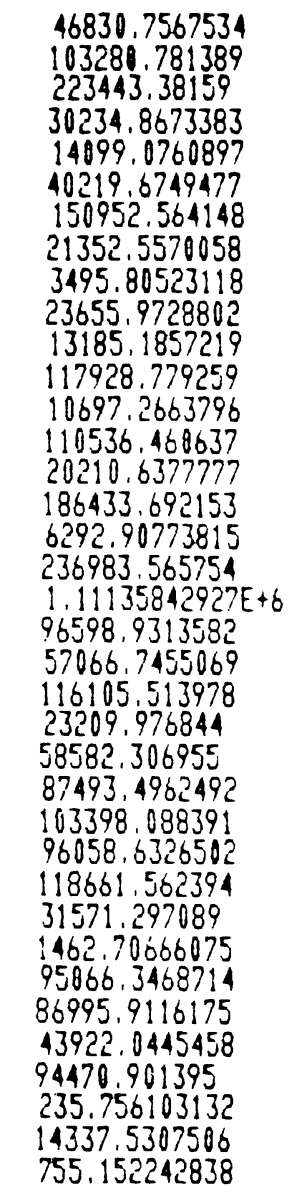 \\
\hline
\end{tabular}

En el caso General, hay dos municipios que incrementarían notablemente sus niveles de accesibilidad: Leganés (nodo 18) y Fuenlabrada (nodo 14). La razón es que la nueva vía les beneficiaría extraordinariamente, al comunicarles con cuatro de los seis ejes radiales. Con incrementos importantes se encuentran, además, otros municipios próximos al trazado del Distribuidor como Coslada (nodo 12) y Navalcarnero (nodo 23).

Desde el punto de vista zonal los incrementos significativos se concentran en la mitad sureste de la provincia. Es de destacar que la otra mitad no percibe apenas beneficio, como se comprueba en la Figura 8.

\begin{tabular}{|c|c|c|}
\hline Nodo & $\begin{array}{l}\text { Accesibilidad } \\
\text { Sit. Inicial }\end{array}$ & $\begin{array}{c}\text { Accesibilidad con } \\
\text { Distribuidor Sur }\end{array}$ \\
\hline $\begin{array}{l}38 \\
39 \\
40 \\
41 \\
42 \\
43 \\
44 \\
45 \\
46 \\
47 \\
48 \\
49 \\
50 \\
51 \\
52 \\
53 \\
54 \\
55 \\
56 \\
57 \\
58 \\
59 \\
60 \\
61 \\
62 \\
63 \\
64 \\
65 \\
66 \\
67 \\
68 \\
69 \\
70 \\
71 \\
72 \\
73 \\
74\end{array}$ & $\begin{array}{l}8247.00094173 \\
18680.0251301 \\
10611.9738168 \\
411.503694523 \\
1258.14235969 \\
2708.54742128 \\
2511.12366628 \\
7979.36738888 \\
21362.0034101 \\
59728.7946642 \\
23859.9018211 \\
135003.472362 \\
254930.14171 \\
10156.7282565 \\
4580.94862646 \\
4020.52498522 \\
16063.59125 \\
33711.053907 \\
159952.624445 \\
99157.2624542 \\
424287.276613 \\
46302.6819082 \\
232280.261634 \\
133739.963886 \\
23252.838787 \\
14776.4688574 \\
6074.69615928 \\
6540.25329274 \\
4931.1875149 \\
8323.31307863 \\
16476.1186487 \\
6121.3647588 \\
27251.9046844 \\
0 \\
0\end{array}$ & 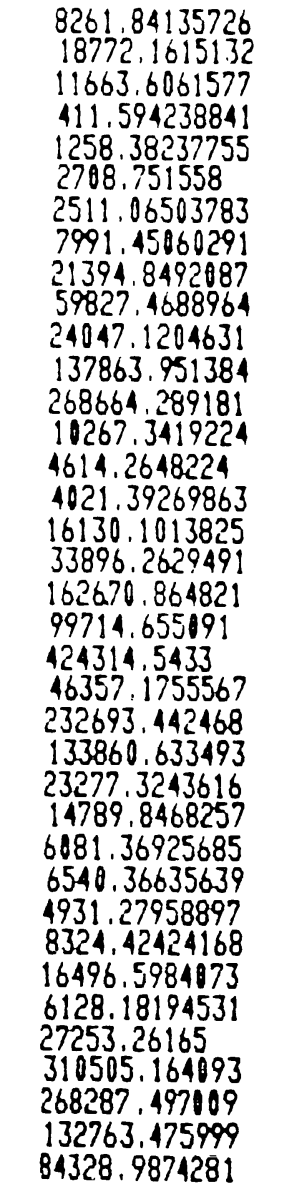 \\
\hline
\end{tabular}

En el caso del Empleo, aunque no hay incrementos "punta" tan acusados, hay un aumento medio de la accesibilidad superior al caso General. Esto es debido a que el Distribuidor Sur comunicaría dos zonas complementarias desde el punto de vista de los puestos de trabajo: cfr. Anexo II. El Corredor Madrid-Alcalá que tiene superávit de puestos de trabajo, y la zona Sur de la de la Corona Metropolitana con un déficit muy importante.

Las zonas donde se localizan los mayores incrementos son las mismas, según se detalla en la Figura 9, donde se daban en el caso General. 


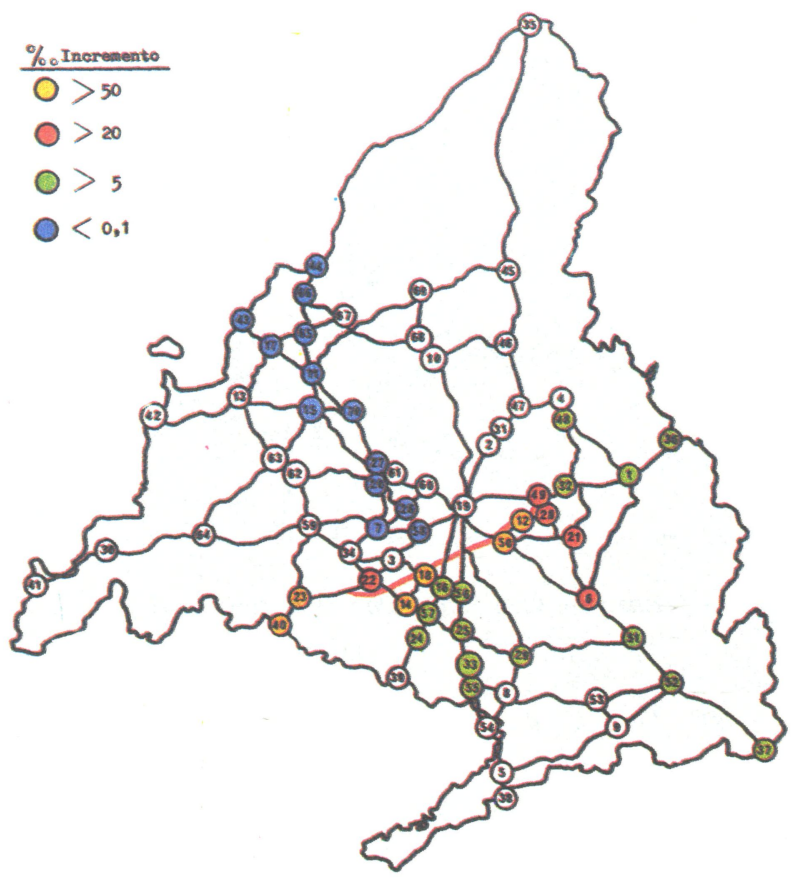

Fig. 8. - Incremento de Accesibilidad Individual General con el Distribuidor Sur.

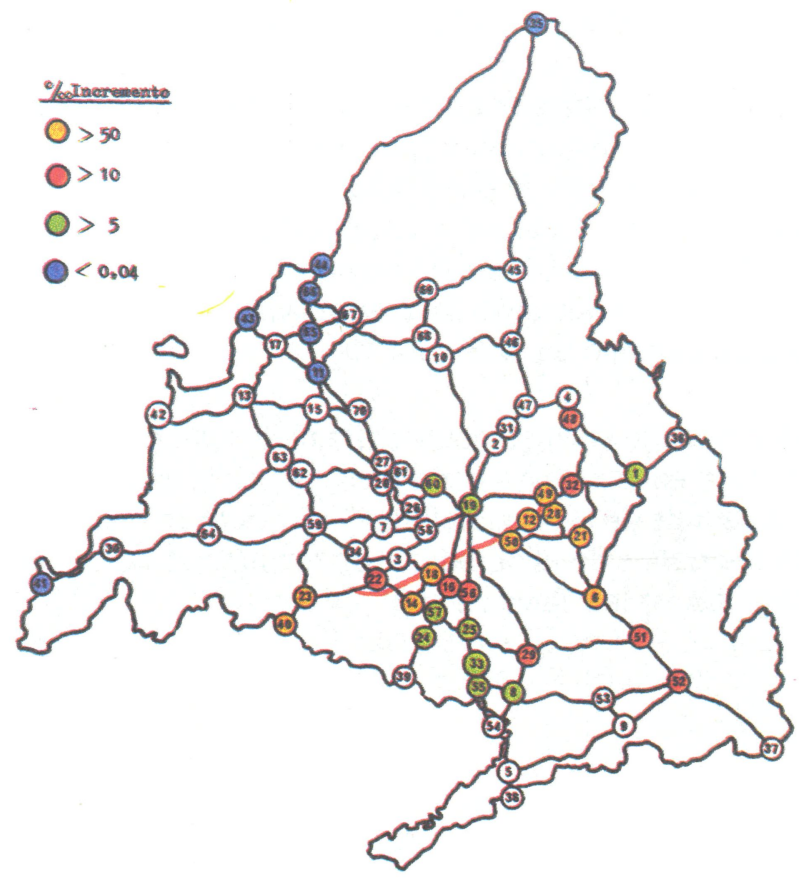

Fig. 9. - Incremento de Accesibilidad Individual al Empleo con el Distribuidor Sur.

TABLA 7

ACCESIBILIDAD INDIVIDUAL AL EMPLEO CON EL DISTRIBUIDOR SUR. INCREMENTOS RESPECTO A LA SITUACION INICIAL

\begin{tabular}{|c|c|c|}
\hline Nodo & $\begin{array}{l}\text { Accesibilidad } \\
\text { Sit. Inicial }\end{array}$ & $\begin{array}{l}\text { Accesibilidad con } \\
\text { Distribuidor Sur }\end{array}$ \\
\hline & 227577.88484 & 229719.917229 \\
\hline ? & 357444.520518 & 358838.644966 \\
\hline 3 & 845542.63253 & 848948.550708 \\
\hline 4 & 113438.665785 & 113911.579043 \\
\hline 5 & 101793.780612 & 101952.001805 \\
\hline 6 & 118148.055839 & 128727.696698 \\
\hline 7 & 503426.294958 & 503488.861696 \\
\hline 8 & 93292.412942 & 93844.626974 \\
\hline 9 & 19108.8826512 & 19114.7526607 \\
\hline 10 & 83327.009248 & 83581.692817 \\
\hline 11 & 58949,5820885 & 58951.8868576 \\
\hline 12 & 347184,287513 & 411986.632981 \\
\hline 13 & 53366.5114364 & 53379,3332273 \\
\hline 14 & 424418,439815 & 514156,636473 \\
\hline 15 & 78000,9442821 & 78006.6130993 \\
\hline 16 & 722257.61383 & $\begin{array}{l}739740.510468 \\
465928540088\end{array}$ \\
\hline 18 & $\begin{array}{r}40384.8081416 \\
617836,216619\end{array}$ & 928637,265708 \\
\hline 19 & $3.17291562225 E+6$ & $3.19168574875 \mathrm{E}+6$ \\
\hline 20 & 329042,013127 & 329079.886095 \\
\hline 21 & 183196.091622 & 199152.517122 \\
\hline 22 & 535274.960226 & 561338.387384 \\
\hline 24 & $\begin{array}{l}131718.965577 \\
307412.244715\end{array}$ & 309288.839106 \\
\hline 25 & 350148.676286 & $353^{5} 22.17105$ \\
\hline 26 & 358764.800335 & 358806.689673 \\
\hline 27 & 320463.834689 & 320501.694794 \\
\hline 28 & 373584.882564 & 402666.550924 \\
\hline 30 & 119064,989381 & $\begin{array}{l}120358,12905 \\
16897,4998476\end{array}$ \\
\hline 31 & 334127.543371 & 335396.565682 \\
\hline 32 & 288185.257001 & 297807.999977 \\
\hline 33 & 181156.285501 & 182635.539486 \\
\hline 34 & 408763.612249 & 409681.501532 \\
\hline 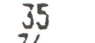 & 45419,3803012 & 45420,1998352 \\
\hline 78 & 132479.246972 & 133063,0189 \\
\hline & 38899,8927124 & 38935.1398124 \\
\hline
\end{tabular}

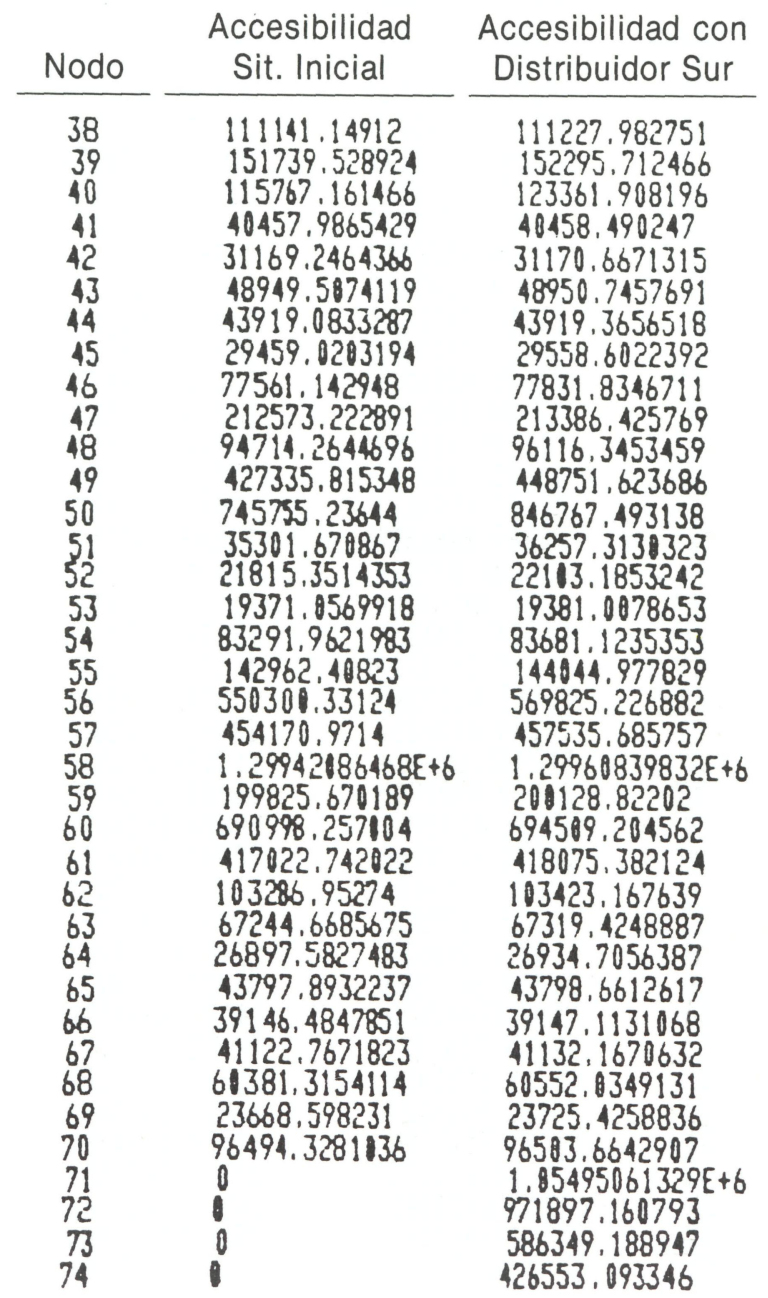




\section{Plan estratégico de la zona oeste}

Dentro de las propuestas del Plan "Estrategia de Transportes en la Region Metropolitana de Madrid" (13) se incluyen dos ejes perpendiculares que vertebrarán toda la zona oeste del Area Metropolitana, con el municipio de Pozuelo (nodo 26) como centro. El esquema consiste en dotar a esa zona de las vías de doble carril que se señalan en la Figura 10.

Modificando las distancias entre nodos del grafo al introducir estas nuevas carreteras, se calculan de nuevo los valores de la Accesibilidad Individual de cada uno de los nodos considerados. Los resultados vienen recogidos en las Tablas 8 y 9.

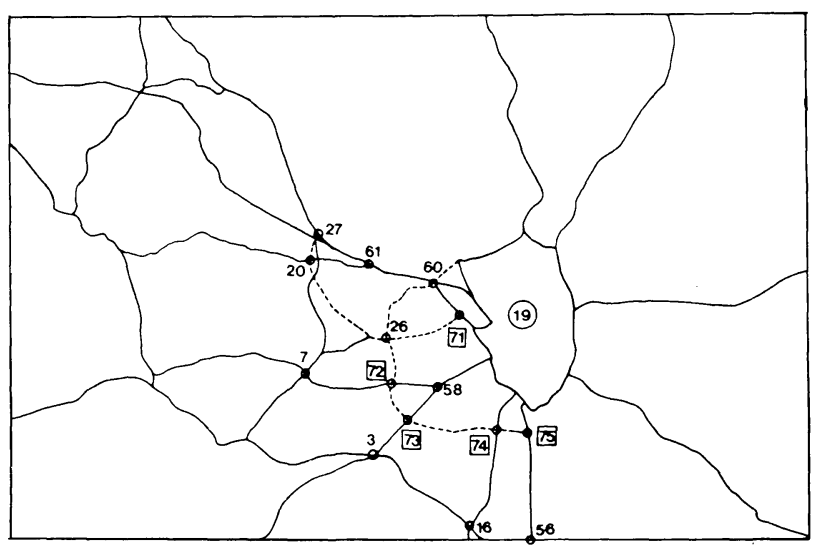

Fig. 10.- Trazado del Plan Estratégico de la Zona Oeste.

TABLA 8

ACCESIBILIDAD INDIVIDUAL GENERAL CON EL PLAN ESTRATEGICO DE LA ZONA OESTE. INCREMENTOS RESPECTO A LA SITUACION INICIAL

\begin{tabular}{|c|c|c|}
\hline Nodo & $\begin{array}{l}\text { Accesibilidad } \\
\text { Sit. Inicial }\end{array}$ & $\begin{array}{l}\text { Accesibilidad } \\
\text { con Plan Oeste }\end{array}$ \\
\hline $\begin{array}{l}1 \\
2 \\
3 \\
1 \\
5 \\
6 \\
7 \\
8 \\
9 \\
10 \\
11 \\
12 \\
13 \\
14 \\
15 \\
16 \\
17 \\
18 \\
19 \\
20 \\
21 \\
22 \\
23 \\
24 \\
25 \\
26 \\
27 \\
28 \\
29 \\
30 \\
31 \\
32 \\
33 \\
34 \\
35 \\
36 \\
37 \\
38\end{array}$ & 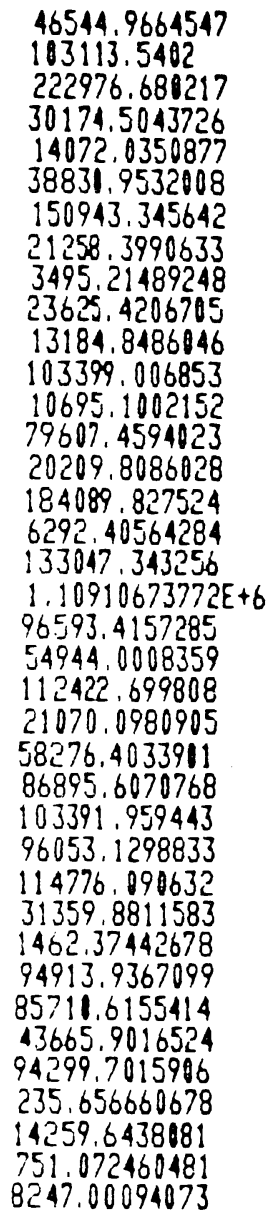 & $\begin{array}{l}46550.8552596 \\
103156.848766 \\
226647.600052 \\
30184.0598136 \\
14089.248127 \\
38845.5651433 \\
155714.038118 \\
21321.5588111 \\
3497.789423 \\
23631.1784775 \\
14578.8575696 \\
103442.903554 \\
11531.2727809 \\
79942.3364877 \\
23642.180552 \\
184859.931907 \\
6570.4131456 \\
134390.720174 \\
1.10969775141 E+6 \\
131732.283171 \\
54963.7205627 \\
113193.270166 \\
21109.1754929 \\
58424.0634075 \\
87241.3402134 \\
274543.588039 \\
120395.437727 \\
114824.60399 \\
31427,6994031 \\
1462.76364558 \\
94952.9964843 \\
85737.21865662 \\
13821.2495646 \\
94398.0294285 \\
235.678673559 \\
14261.2486946 \\
751.192012884 \\
8256.14765699\end{array}$ \\
\hline
\end{tabular}

(c) Consejo Superior de Investigaciones Científicas Licencia Creative Commons 3.0 España (by-nc)

\begin{tabular}{|c|c|c|}
\hline Nodo & $\begin{array}{c}\text { Accesibilidad } \\
\text { Sit. Inicial }\end{array}$ & $\begin{array}{l}\text { Accesibilidad } \\
\text { con Plan Oeste }\end{array}$ \\
\hline $\begin{array}{l}39 \\
10 \\
41 \\
42 \\
13 \\
14 \\
45 \\
46 \\
17 \\
48 \\
19 \\
50 \\
51 \\
52 \\
53 \\
54 \\
55 \\
56 \\
57 \\
58 \\
59 \\
60 \\
61 \\
62 \\
63 \\
64 \\
65 \\
66 \\
67 \\
68 \\
69 \\
70 \\
71 \\
72 \\
73 \\
74 \\
75\end{array}$ & $\begin{array}{l}18680.0251301 \\
10601.9738168 \\
411.503694523 \\
1258.14235969 \\
2708.54742128 \\
2511.02366628 \\
7979.36730888 \\
21362.0034101 \\
59728.7946642 \\
23859.9018211 \\
135003.472362 \\
254931.14171 \\
10156.7282565 \\
4580.94862646 \\
4020.52498522 \\
16063.59125 \\
33711.053907 \\
159952.624445 \\
99157.2624542 \\
424287.276613 \\
46302.6819082 \\
232281.260634 \\
133739.963886 \\
23252.838787 \\
14776.4088574 \\
5074.69615928 \\
6540.25329274 \\
4931.1875149 \\
8323.31307863 \\
16476.1186487 \\
6121.3647588 \\
27251.9046844 \\
0 \\
0 \\
0\end{array}$ & 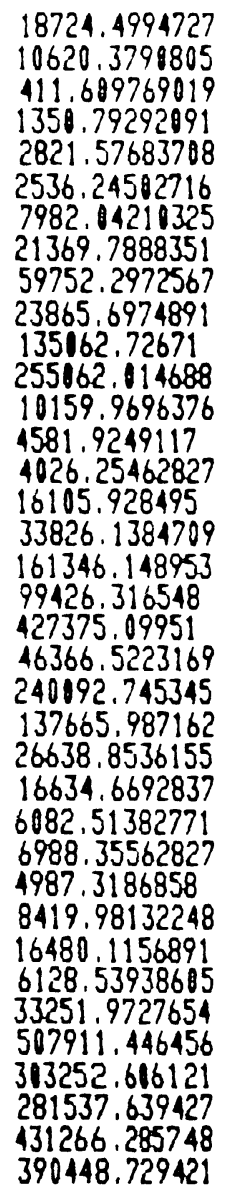 \\
\hline
\end{tabular}

http://informesdelaconstruccion.revistas.csic.es 
TABLA 9

ACCESIBILIDAD INDIVIDUAL AL EMPLEO CON EL PLAN ESTRATEGICO DE LA ZONA OESTE. INCREMENTOS RESPECTO A LA SITUACION INICIAL

\begin{tabular}{|c|c|c|}
\hline Nodo & $\begin{array}{l}\text { Accesibilidad } \\
\text { Sit. Inicial }\end{array}$ & $\begin{array}{l}\text { Accesibilidad } \\
\text { con Plan Oeste }\end{array}$ \\
\hline $\begin{array}{l}1 \\
2 \\
3 \\
4 \\
5 \\
6 \\
7 \\
8 \\
9 \\
10 \\
11 \\
12 \\
13 \\
14 \\
15 \\
16 \\
17 \\
18 \\
19 \\
20 \\
21 \\
22 \\
23 \\
24 \\
25 \\
26 \\
27 \\
28 \\
29 \\
30 \\
31 \\
32 \\
33 \\
34 \\
35 \\
36 \\
37 \\
38\end{array}$ & 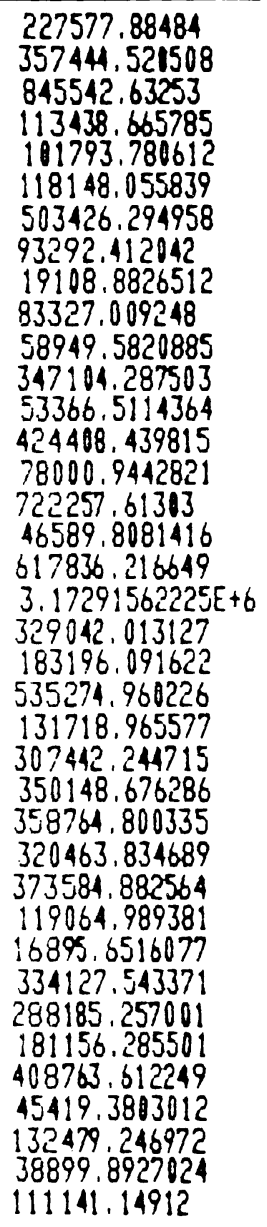 & 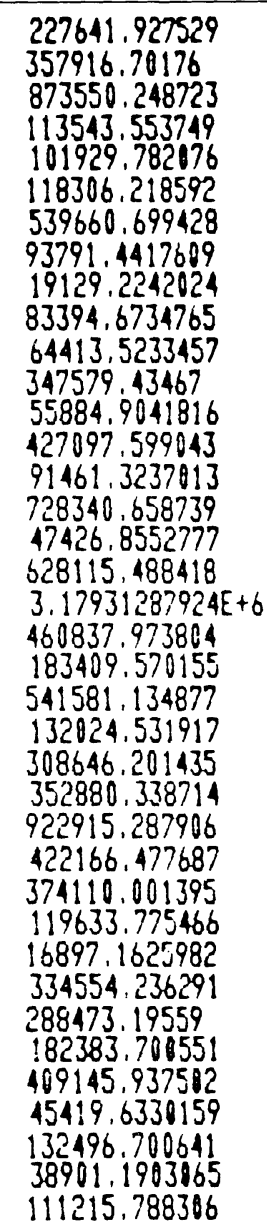 \\
\hline
\end{tabular}

Con este plan los incrementos medios son similares en los dos casos estudiados: desplazamientos por todos los motivos y sólo al puesto de trabajo. El punto que obtiene un incremento espectacular es Pozuelo de Alarcón (nodo 26) que duplica ampliamente su accesibilidad en ambos casos. Le siguen, a notable distancia, los nodos situados sobre la N-VI y puntos cercanos a ambos lados. Podemos destacar Las Rozas (nodo 27), Torrelodones (nodo 70), Galapagar (nodo 15), Valdemorillo (nodo 63), etc.

\begin{tabular}{|c|c|c|}
\hline Nodo & $\begin{array}{l}\text { Accesibilidad } \\
\text { Sit. Inicial }\end{array}$ & $\begin{array}{l}\text { Accesibilidad } \\
\text { con Plan Oeste }\end{array}$ \\
\hline $\begin{array}{l}39 \\
40 \\
41 \\
42 \\
43 \\
44 \\
45 \\
46 \\
47 \\
48 \\
49 \\
50 \\
51 \\
52 \\
53 \\
54 \\
55 \\
56 \\
57 \\
58 \\
59 \\
60 \\
61 \\
62 \\
63 \\
64 \\
65 \\
66 \\
67 \\
68 \\
69 \\
70 \\
71 \\
72 \\
73 \\
74 \\
75\end{array}$ & $\begin{array}{l}151739.528924 \\
115767.161466 \\
40457.9865429 \\
31169.2464366 \\
48949.5074119 \\
43919.0833287 \\
29459.0203194 \\
77561.142948 \\
212573.222891 \\
94714.2644696 \\
427335.815348 \\
745755.23644 \\
35301.670867 \\
21815.3514353 \\
19371.8569918 \\
83291.9621983 \\
142962.40823 \\
550310.33124 \\
454170,9714 \\
1.299421864686+6 \\
199825.670189 \\
690998.257104 \\
417022.742022 \\
103286.95274 \\
67244.6685675 \\
26897.5827483 \\
43797.8932237 \\
39146.4847851 \\
41122.7671823 \\
60381.3154114 \\
23668.598231 \\
96494.3281136 \\
0 \\
1 \\
0\end{array}$ & 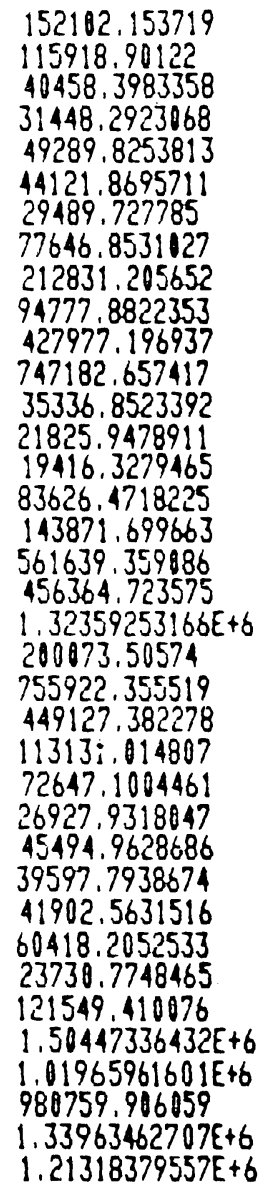 \\
\hline
\end{tabular}

Las mejoras, en este caso, se encuentran muy localizadas sobre el eje de la N-VI, como se puede comprobar en las Figuras 11 y 12. En el caso del Empleo, la influencia de la construcción de las nuevas vias se prolonga más a ambos lados por los municipios de la Sierra en el norte y por los del sur de la Corona Metropolitana y N-IV. El resto de la provincia no recibe apenas beneficio con este plan, por lo que se puede decir que se trata de una alternativa con efectos muy localizados. 


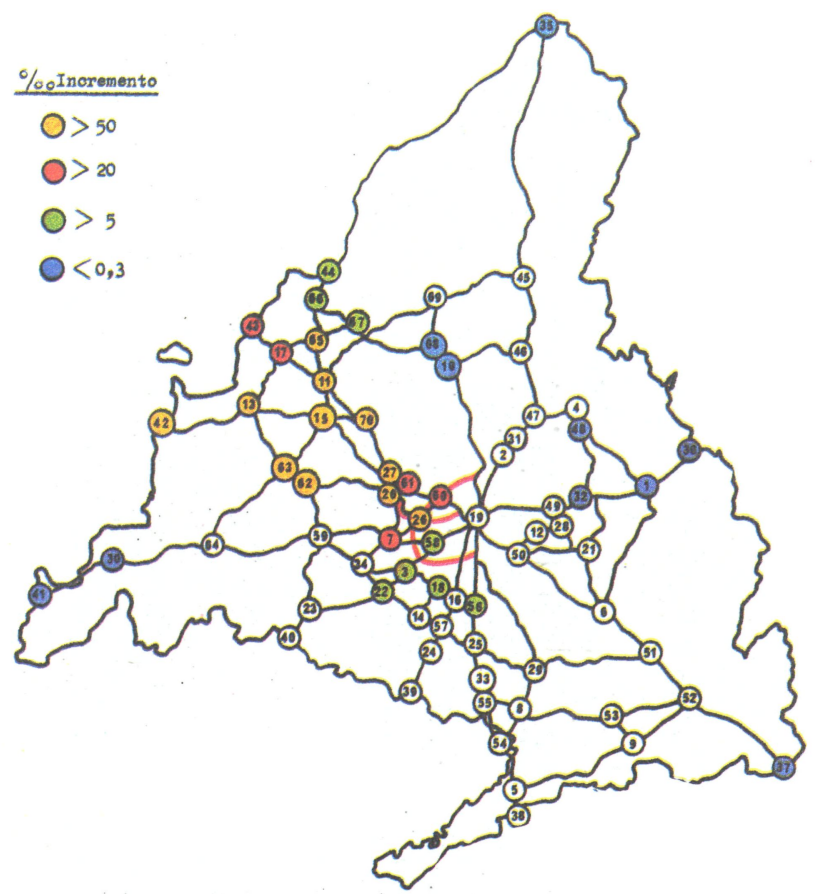

Fig. 11.- Incremento de Accesibilidad Individual General con el Plan Estratégico de la Zona Oeste.

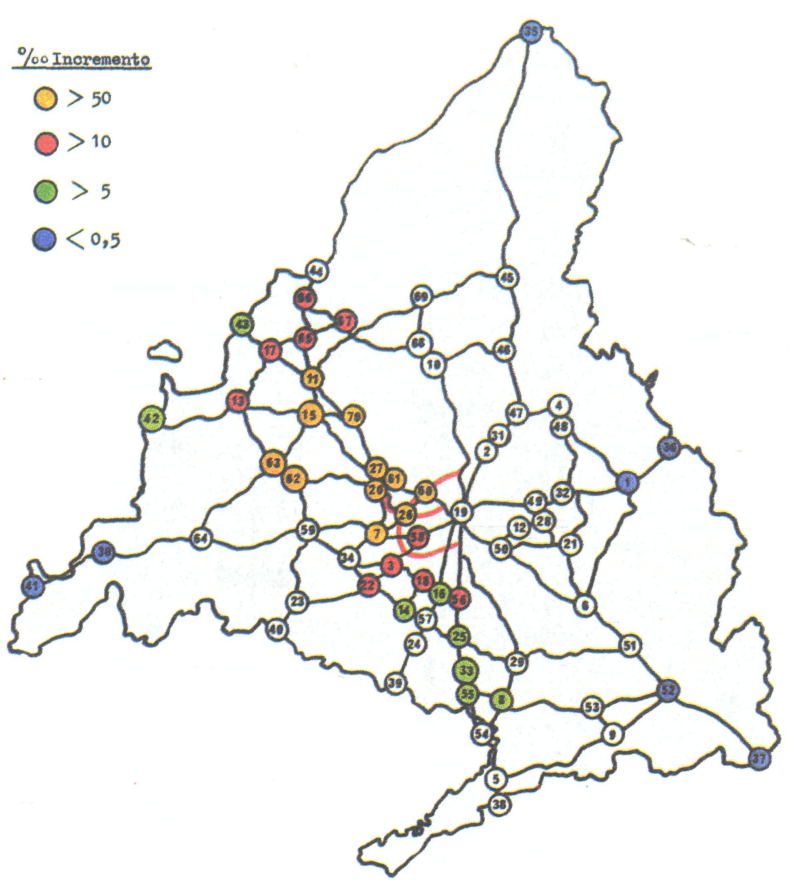

Fig. 12.-Incremento de Accesibilidad Individual al Empleo con el Plan Estratégico de la Zona Oeste.

\section{ANALISIS DE RESULTADOS Y CONCLUSIONES}

Podemos analizar, también, el aumento global de Accesibilidad Individual obtenido por todos los nodos del gra inicial. Sumaremos los valores obtenidos por cada uno, en el caso General y en el del Empleo, en la Situación Inicial y con cada uno de los tres planes de transporte descritos. Los resultados son los que recoge la tabla siguiente:

\begin{tabular}{|l|c|c|}
\hline \multirow{2}{*}{ A. Cinturón de Circunvalación } & \multicolumn{2}{|c|}{ Incrementos Globales $(\%$ ) $)$} \\
\cline { 2 - 3 } \cline { 2 - 3 } B. Distribuidor Sur & 5,93 & 3,95 \\
C. Plan de la Zona Oeste & 41,68 & 69,51 \\
& 58,43 & 53,16 \\
\hline
\end{tabular}

Del estudio de esta Tabla de "Accesibilidades Globales" cabe señalar que, en el caso General, la propuesta más beneficiosa sería el Plan Estratégico de la Zona Oeste, aunque con el Distribuidor Sur se obtiene un incremento medio de accesibilidad también importante.

En el caso del Empleo la situación es contraria. Los mejores resultados corresponden al Distribuidor Sur, y el Plan de la Zona Oeste queda en segundo lugar.
Esto nos permite la toma de decisiones con una visión general, eligiendo las alternativas que más benefician a un mayor número de usuarios, considerados individualmente.

Cabe, sin embargo, tener en cuenta los resultados del apartado anterior para diseñar políticas de equilibrio regional en el ámbito provincial. Si queremos contribuir a la homogeneización de los niveles de accesibilidad y propiciar a una mayor igualdad de oportunidades, no cabe duda que lo mejor será construir el Cinturón de Circunvalación. Si queremos mejorar el sureste de la provincia, habrá que optar por el Distribuidor Sur. En cambio, si se pretende mejorar la comunicación con la zona de la Sierra - segundas residencias, deportes de montaña, etc.- habremos de recurrir al Plan Estratégico de la Zona Oeste.

Este análisis confirma las posibilidades de los indicadores de accesibilidad en la planificación del transporte, y concretamente en el campo de las nuevas infraestructuras. Los niveles de accesibilidad que se obtendrían en cada caso, permiten entrar en el proceso de decisión con análisis de tipo coste/beneficios o de tipo multicriterio. 
ANEXO I

RELACION DE NODOS Y VARIABLES CONSIDERADAS

\begin{tabular}{|c|c|}
\hline Nodo & Nombre \\
\hline 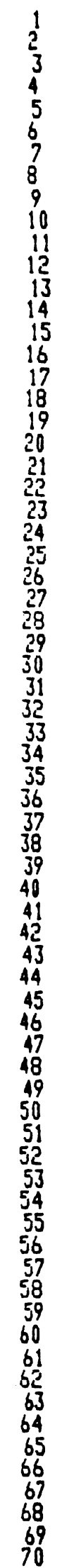 & 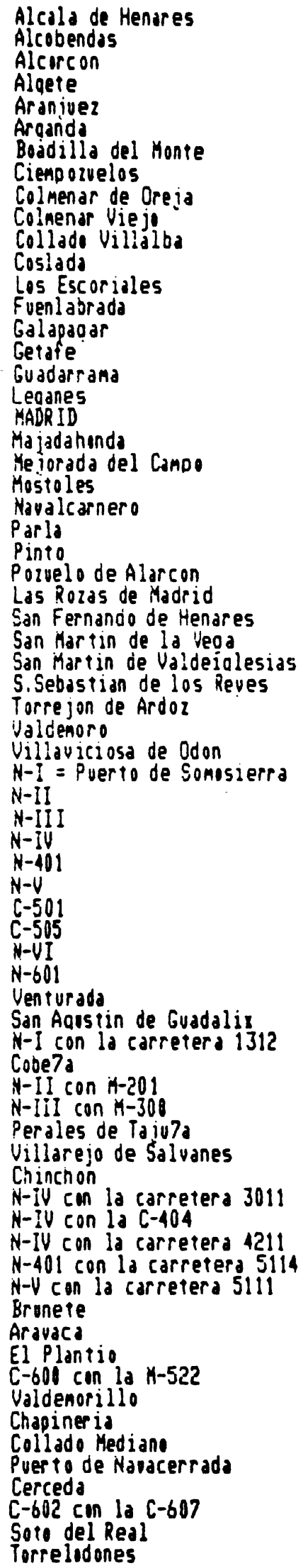 \\
\hline
\end{tabular}

\begin{tabular}{|c|c|c|}
\hline Población & Pobl. activa & Puest. de Trabajo \\
\hline 147969 & 37038 & 27714 \\
\hline $\begin{array}{r}69979 \\
136997\end{array}$ & 18756 & $\begin{array}{l}12994 \\
16478\end{array}$ \\
\hline $\begin{array}{l}136997 \\
7501\end{array}$ & $\begin{array}{l}09019 \\
1597\end{array}$ & $\begin{array}{r}16478 \\
1724\end{array}$ \\
\hline 37304 & 9085 & 8484 \\
\hline $\begin{array}{r}24214 \\
8320\end{array}$ & $\begin{array}{l}6494 \\
1718\end{array}$ & $\begin{array}{l}8294 \\
1913\end{array}$ \\
\hline 9826 & 2283 & 2259 \\
\hline 5147 & 1313 & 1183 \\
\hline 31097 & 5640 & $\begin{array}{l}7149 \\
4691\end{array}$ \\
\hline $\begin{array}{l}20404 \\
6.5098\end{array}$ & 16473 & 9327 \\
\hline & 4228 & 3702 \\
\hline 118224 & $\begin{array}{r}24029 \\
1656\end{array}$ & 17581 \\
\hline 132937 & 33014 & 24514 \\
\hline 5844 & 1770 & 1343 \\
\hline 164989 & $\begin{array}{r}44936 \\
040500\end{array}$ & $\begin{array}{r}18631 \\
1007005\end{array}$ \\
\hline 3068363 & 969589 & 1193295 \\
\hline $\begin{array}{l}29174 \\
11524\end{array}$ & $\begin{array}{l}7444 \\
2690\end{array}$ & $\begin{array}{l}3846 \\
2649\end{array}$ \\
\hline 176148 & 43477 & 18846 \\
\hline 8994 & 2112 & 2068 \\
\hline $\begin{array}{l}638 / 8 \\
20017\end{array}$ & $\begin{array}{l}14988 \\
5374\end{array}$ & 4602 \\
\hline 36937 & 8630 & 2792 \\
\hline $\begin{array}{l}21121 \\
20015 \\
32015\end{array}$ & $\begin{array}{l}1076 \\
5054\end{array}$ & $\begin{array}{l}4855 \\
5256\end{array}$ \\
\hline 5590 & 1433 & 1585 \\
\hline 5041 & $\begin{array}{r}1272 \\
11810\end{array}$ & 1159 \\
\hline $\begin{array}{l}47320 \\
75465\end{array}$ & 22252 & 23503 \\
\hline 15971 & 3563 & 3671 \\
\hline $\begin{array}{r}3033 \\
45180\end{array}$ & 1632 & $\begin{array}{r}1847 \\
170\end{array}$ \\
\hline 76110 & 0 & 1701 \\
\hline 36780 & 0 & 193 \\
\hline $\begin{array}{l}799100 \\
6=0,010\end{array}$ & 0 & $\begin{array}{r}750 \\
1240\end{array}$ \\
\hline 66840 & 0 & 183 \\
\hline 38730 & 0 & 14 \\
\hline $\begin{array}{l}25,570 \\
35950\end{array}$ & 0 & 180 \\
\hline 32990 & 0 & 370 \\
\hline 280 & $\begin{array}{r}59 \\
570\end{array}$ & 64 \\
\hline $\begin{array}{r}2474 \\
0\end{array}$ & 3 & $\begin{array}{l}570 \\
0\end{array}$ \\
\hline $83 i$ & $18 i$ & $19 !$ \\
\hline & 0 & 0 \\
\hline 1879 & 532 & 432 \\
\hline 4594 & 1264 & 1056 \\
\hline 4090 & $\begin{array}{r}1087 \\
0\end{array}$ & 940 \\
\hline & 0 & 0 \\
\hline & $y$ & 0 \\
\hline & 80 & 20 \\
\hline $\begin{array}{l}1610 \\
8904\end{array}$ & 2813 & $\begin{array}{l}370 \\
2642\end{array}$ \\
\hline 2086 & 659 & 617 \\
\hline 2355 & 624 & 541 \\
\hline $\begin{array}{r}768 \\
1697\end{array}$ & $\begin{array}{l}200 \\
457\end{array}$ & $\begin{array}{l}177 \\
390\end{array}$ \\
\hline 0 & 0 & 370 \\
\hline 754 & $\begin{array}{c}398 \\
0\end{array}$ & $\begin{array}{c}174 \\
0\end{array}$ \\
\hline 1714 & 398 & 394 \\
\hline & & \\
\hline
\end{tabular}


ANEXO II

DESEQUILIBRIOS ABSOLUTOS RESIDENCIA/EMPLEO PARA ACTIVOS DEL AREA METROPOLITANA

\begin{tabular}{|c|c|c|}
\hline & $\begin{array}{l}\text { Saldo numérico } \\
\text { empleos-activos }\end{array}$ & $\begin{array}{l}\text { Población que } \\
\text { trabaja fuera } \\
\text { de su zona } \\
\text { de residencla }\end{array}$ \\
\hline 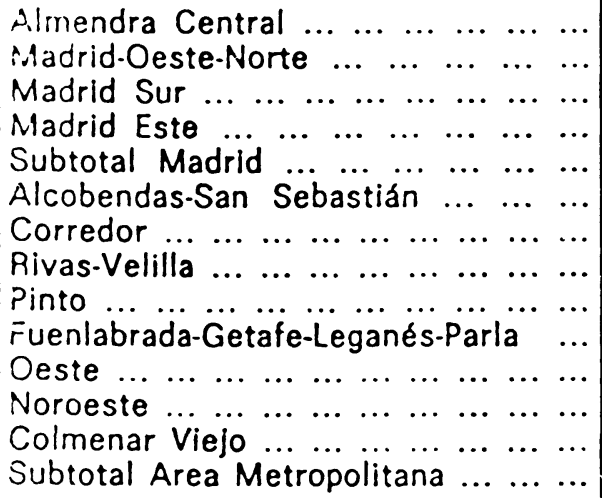 & $\begin{array}{r}343.749 \\
-78.430 \\
-142.838 \\
-20.709 \\
-5.261 \\
4.831 \\
-232 \\
-395 \\
-41.365 \\
-64.725 \\
4.754 \\
-498\end{array}$ & $\begin{array}{r}109.608 \\
175.509 \\
226.151 \\
132.737 \\
644.005 \\
13.739 \\
24.862 \\
412 \\
4.085 \\
71.316 \\
70.329 \\
9.578 \\
1.545 \\
195.866\end{array}$ \\
\hline $\begin{array}{lllllllll}\text { TOTAL } & \ldots & \ldots & \ldots & \ldots & \ldots & \ldots & \ldots & \ldots\end{array}$ & & 839.871 \\
\hline
\end{tabular}

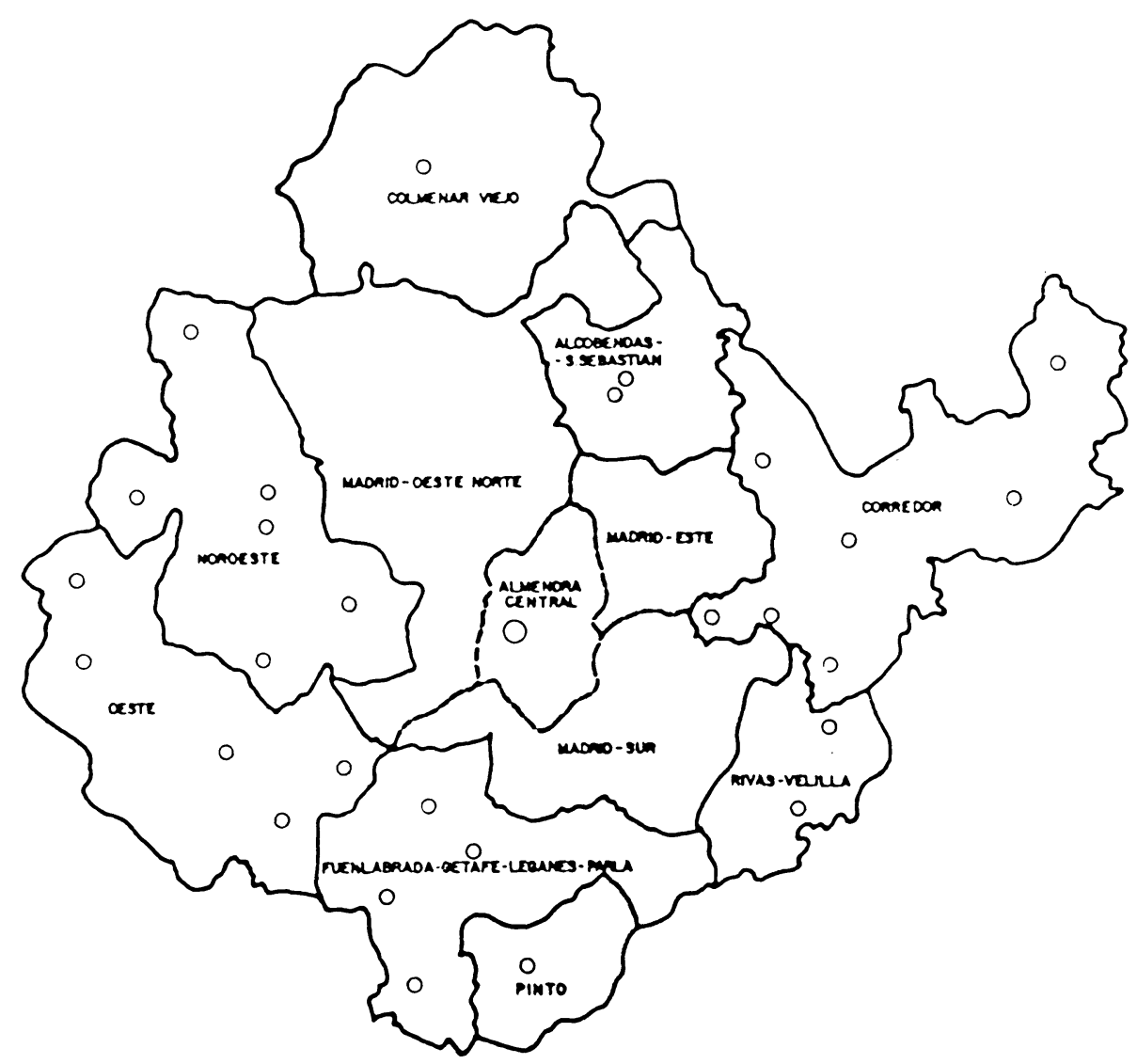

Desequilibrios Población/Empleo en el AREA METROPOLITANA MADRILEÑA. Fuente: De la Paz (14). 
(1) DALVI, M.Q. Behauvioral modelling accesibility, movility and need: concepts and measurement. Hensher and Stopher, eds. Beahuvioral Travel Modelling. London: Croom Helm. 1978.

(2) MORRIS, J.M. et al. Accesibility Indicators for Transport Planing. Transport Research-A, Vol. 13-A, 1979.

(3) POULIT, J. Urbanisme et transport: les critères d'accesibilitè et de dèveloppement urbain. SETRA. Ministère des Transports. Paris, 1974.

(4) MOPU. Avance del Plan General de Carreteras. Estudio sobre la accesibilidad de la Red. MOPU. Madrid, 1982.

(5) CARROTHERS, Gerald A. P. An Historical Review of the Gravity and Potential concepts of the Human Interaction. Journal of the American Institute of Planners, Vol. 22, 1956.

(6) HANSEN, Walter G. How Accesibility Shapes land Use. Journal of the American Institute of Planners, Vol. 25, 1959.

(7) KOENING, J. G. La thèorie de l'accesibilitè urbaine, un nouvel outil an service de l'amènageur. Revue Générale des routes et des Arerodromes, 1979.

(8) MONZON DE CACERES, Andrés. El Concepto de Distancia Operacional del Usuario en las Areas Metropolitanas. Actas del Congreso Europeo de Ordenación del Territorio de Valencia, 1988.

(9) MAPA DE AFOROS. MOPU, 1984.

(10) COMUNIDAD DE MADRID. Municipios de la Comunidad de Madrid. Anuario Estadístico 1985, Vol. II.

(11) COMUNIDAD DE MADRID. Plan de Carreteras 1986-1993. Programa de Actuaciones. Consejería de Obras Públicas y Transportes. Madrid, 1985.

(12) MOPU. Plan General de Carreteras 1984-1991. Madrid, 1984.

(13) COMUNIDAD DE MADRID. Plan Estratégico de Transportes de la Región Metropolitana de Madrid. Madrid, 1988.

(14) DE LA PAZ, José et al. Población y Empleo. Documentación Urbanística. Diputación de Madrid, 1982.

\section{publicación del IETcc / CSIC}

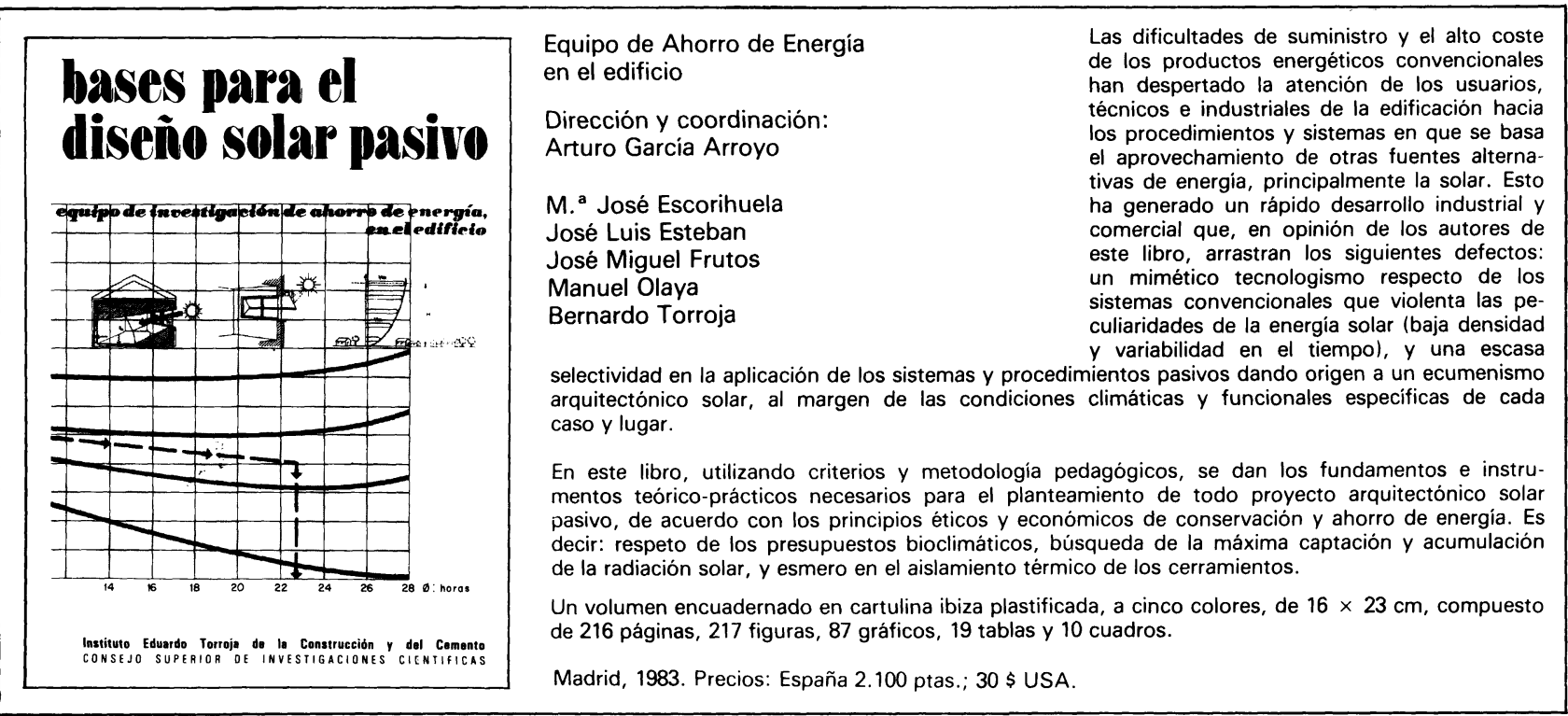

